\title{
Zur Bindung des Wasserstoffs im Gitter kristalliner Aquoxide
}

\author{
E. SCHWARZMANN \\ Anorg.-chemisches Institut der Universität Göttingen \\ (Z. Naturforschg. 24 b, 1104-1120 [1969]; eingegangen am 1. Juni 1969)
}

\begin{abstract}
Aquoxide sind anorganische Verbindungen, die sich experimentell oder formal aus Oxid und Wasser ableiten ${ }^{1}$. Der Wasserstoff kann darin in Form von OH-Gruppen, $\mathrm{H}_{2} \mathrm{O}$-Molekülen, hydratisierten Protonen, direkt an das Zentralatom gebunden oder auf Zwischengitterplätzen eingelagert als feste Lösung von Protonen in einem Oxidgitter vorliegen. Es wird eine Systematik dieser Verbindungen gegeben. Eine der Methoden zur Festlegung des Bindungstyps ist die IR-Spektroskopie. An Hand von IR-Spektren kristalliner Hydroxide wird über Ergebnisse und Probleme bei der Bestimmung der Bindung des Wasserstoffs in Kristallgittern berichtet.
\end{abstract}

\section{Bindungstypen}

Beim strukturellen Aufbau kristalliner Aquoxide kann zwischen den folgenden Bindungstypen unterschieden werden:

\section{Hydroxide}

a) Hydroxide ohne $\mathrm{O}-\mathrm{H} \cdots \mathrm{O}$-Bindungen

a) Hydroxide mit Koordinationsgruppen $\mathrm{M}(\mathrm{OH})_{\mathrm{m}}$

Diese Verbindungen kristallisieren in einem Ionengitter, das aus Metall- und Hydroxyl-Ionen aufgebaut ist. $\mathrm{Ca}(\mathrm{OH})_{2}(\mathrm{P} \overline{3} \mathrm{~m} ; Z=1)$ besitzt ein Schichtengitter vom $\mathrm{CdJ}_{2}$-Typ. Jedes $\mathrm{Ca}^{2 \oplus}$-Ion ist von sechs $\mathrm{OH}^{\ominus}$-Ionen, jedes $\mathrm{OH}^{\ominus}$-Ion auf der einen Seite von

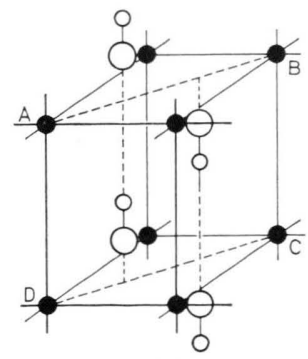

(a)

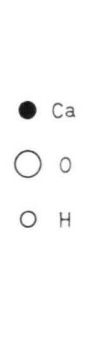

Abb. 1. Kristallstruktur von $\mathrm{Ca}(\mathrm{OH})_{2}$. a) Einheitszelle b) Ausschnitt parallel der (110)-Ebene. $\mathrm{Ca}^{2} \oplus$-Ionen sitzen auf den Ecken der Zelle, $\mathrm{OH}^{\ominus}$.Ionen auf den Linien $\frac{1}{3} \frac{2}{3} z$ und $\frac{2}{3} \frac{1}{3} z$. Nach Busing und Levy ${ }^{7}$

drei $\mathrm{OH}^{\ominus}$-Ionen, auf der anderen Seite von drei $\mathrm{Ca}^{2}{ }^{\oplus}$-Ionen umgeben. Die Umgebung von $\mathrm{OH}$ zeigt, daß keine $\mathrm{O}-\mathrm{H} \cdots \mathrm{O}$-Bindung vorhanden ist. Die $\mathrm{O}-\mathrm{H}$-Bindungen liegen senkrecht zu den Schichten ${ }^{2-10}$ (Abb. 1). Isostrukturell mit $\mathrm{Ca}(\mathrm{OH})_{2}$ sind $\mathrm{Mg}(\mathrm{OH})_{2}{ }^{4,5,11-14}, \mathrm{Mn}(\mathrm{OH})_{2}{ }^{15}, \mathrm{Fe}(\mathrm{OH})_{2}{ }^{16}$,
$\mathrm{Co}(\mathrm{OH})_{2}{ }^{17,18}, \mathrm{Ni}(\mathrm{OH})_{2}{ }^{17,19-22}$ und das nur durch Verunreinigungen stabilisierte $\alpha-\mathrm{Zn}(\mathrm{OH})_{2}{ }^{17}$. Weiterhin ist von den folgenden Hydroxiden mit Koordinationsgruppen $\mathrm{M}(\mathrm{OH})_{m}$ ohne $\mathrm{O}-\mathrm{H} \cdots \mathrm{O}$-Bindungen eine Kristallstrukturanalyse durchgeführt worden: $\mathrm{Li}(\mathrm{OH})^{23-26}, \alpha-\mathrm{Na}(\mathrm{OH})^{27-29}, \quad \beta-\mathrm{Na}(\mathrm{OH})^{28}$, $\alpha-\mathrm{Cd}(\mathrm{OH})_{2}{ }^{20,30}, \beta-\mathrm{Cd}(\mathrm{OH})_{2}{ }^{20,30,31}, \mathrm{Y}(\mathrm{OH})_{3}{ }^{32-37}$ und die damit isostrukturellen Verbindungen $\mathrm{La}(\mathrm{OH})_{3}{ }^{36,37-41}$ und die Hydroxide der Seltenen Erdmetalle ${ }^{33-35,37,39,40,42}$.

B) Oxidhydroxide mit Koordinationsgruppen $\mathrm{M}(\mathrm{OH})_{\mathrm{m}} \mathrm{O}_{\mathrm{n}}$

Die Koordinationspolyeder $\mathrm{M}(\mathrm{OH})_{m} \mathrm{O}_{\mathrm{n}}$ haben O-Atome und OH-Gruppen gemeinsam. $\mathrm{YO}(\mathrm{OH})$ $\left(\mathrm{P} 2_{1} / \mathrm{m} ; Z=2\right)$ kristallisiert in einem Gitter, in dem jedes Y-Atom von sieben O-Atomen bzw. OH-Gruppen, jedes $\mathrm{O}$-Atom von vier $\mathrm{Y}$-Atomen und jede $\mathrm{OH}$ Gruppe von drei Y-Atomen umgeben ist. Es liegt keine Wasserstoffbrückenbindung vor ${ }^{43-45}$. Isostrukturell mit $\mathrm{YO}(\mathrm{OH})$ sind $\mathrm{LaO}(\mathrm{OH})^{46}, \mathrm{HoO}(\mathrm{OH})^{47}$ und $\mathrm{YbO}(\mathrm{OH})^{47}$.

\section{b) Hydroxide mit $\mathrm{O}-\mathrm{H} \cdots O$-Bindungen}

a) Hydroxide mit Koordinationsgruppen $\mathrm{M}(\mathrm{OH})_{m}$

Die Koordinationspolyeder der $\mathrm{Art} \mathrm{M}(\mathrm{OH})_{4}$ und $\mathrm{M}(\mathrm{OH})_{6}$ haben $\mathrm{OH}$-Gruppen gemeinsam. Im Kristallgitter der Tieftemperaturphase von $\mathrm{K}(\mathrm{OH}) \quad\left(\mathrm{P} 2_{1}\right.$; $Z=2$ ) ist jedes $\mathrm{K}$-Atom verzerrt oktaedrisch von O-Atomen umgeben. Diese $\mathrm{KO}_{6}$-Oktaeder haben Kanten derart gemeinsam, daß jedes O-Atom verzerrt oktaedrisch von K-Atomen umgeben ist. Parallel zur b-Achse liegt eine Sauerstoff-SauerstoffZickzack-Kette. Auf Grund stereochemischer Argumente wird angenommen, daß die Wasserstoffatome 
nahezu oder direkt in der Ebene der Zickzack-Sauerstoff-Kette liegen. Nach IR-Messungen befinden sich die Wasserstoffatome in geordneten Lagen entlang der Kette mit nahezu oder exakt linearen $\mathrm{O}-\mathrm{H} \cdots \mathrm{O}$-Bindungen der Länge $[\mathrm{O}(\mathrm{H}) \ldots \mathrm{O}$-Abstand] $3,35 \AA^{48-50}$. Isostrukturell mit $\mathrm{K}(\mathrm{OH})$ ist $\mathrm{Rb}(\mathrm{OH})^{48}$. Auch von den folgenden Hydroxiden sind Kristallstrukturanalysen durchgeführt worden: $\beta$ - $\mathrm{Be}(\mathrm{OH})_{2}{ }^{51}$ und das damit isomorphe $\varepsilon-\mathrm{Zn}(\mathrm{OH})_{2}$ siehe ${ }^{52,53}$, Bayerit $\mathrm{Al}(\mathrm{OH})_{3}{ }^{54-58}$, Hydrargillit $\mathrm{Al}(\mathrm{OH})_{3}$ monoklin ${ }^{59,60}$ und triklin ${ }^{59}$, Nordstrandit $\mathrm{Al}(\mathrm{OH})_{3}{ }^{61}, \mathrm{Ga}(\mathrm{OH})_{3}{ }^{62}$ und die damit isomorphen Verbindungen $\mathrm{In}(\mathrm{OH})_{3}{ }^{63-66}$ und $\mathrm{Sc}(\mathrm{OH})_{3}{ }^{63,64}$, $\mathrm{Sr}(\mathrm{OH})_{2}{ }^{67,68}, \alpha-\mathrm{Ba}(\mathrm{OH})_{2}{ }^{69,70}, \mathrm{Cu}(\mathrm{OH})_{2}{ }^{71,72}$ und $\gamma-\mathrm{Cd}(\mathrm{OH})_{2}{ }^{30,73-75}$.

$\beta$ ) Oxidhydroxide mit Koordinationsgruppen $\mathrm{M}(\mathrm{OH})_{\mathrm{m}} \mathrm{O}_{\mathrm{n}}$

Die Koordinationspolyeder der $\operatorname{Art} \mathrm{M}(\mathrm{OH})_{m} \mathrm{O}_{n}$ haben O-Atome und OH-Gruppen gemeinsam. Bei Diaspor, $\alpha-\mathrm{AlO}(\mathrm{OH}),(\mathrm{Pbnm} ; Z=4)$ befinden sich alle Atome in der Einheitszelle auf zwei Spiegelebenen. Es treten zwei Sorten von O-Atomen auf, $\mathrm{O}_{\mathrm{I}}$ und $\mathrm{O}_{\mathrm{II}}$. Jedes Al-Atom hat drei von jeder Sauerstoff-Sorte als Nachbarn. Die resultierenden $\mathrm{AlO}_{6}$. Oktaeder haben Kanten derart gemeinsam, daß sich "double rutile strings" ausbilden, die sich in der $c$-Richtung erstrecken, und diese "double strings“ werden durch gemeinsame Sauerstoff-Ecken zu einem dreidimensionalen Netzwerk verbunden. Eine Art von $\mathrm{O}_{\mathrm{I}} \ldots \mathrm{O}_{\mathrm{II}}$-Abständen ist kürzer als die anderen und entspricht einer nichtlinearen Wasserstoffbrücke der Länge 2,650 A. Der $\mathrm{O}_{\mathrm{II}} \ldots$. H-Abstand beträgt $0,990 \AA$, der Winkel zwischen dem $\mathrm{O}_{\mathrm{II}}-\mathrm{H}-$ Vektor und der $\mathrm{O}_{\mathrm{II}} \ldots \mathrm{O}_{\mathrm{I}}$-Verbindungslinie $12,1^{\circ}{ }_{76-78}$ (Abb. 2).

Isostrukturell mit $\alpha-\mathrm{AlO}(\mathrm{OH})$ sind $\mathrm{GaO}(\mathrm{OH})^{79}$, $\alpha-\mathrm{ScO}(\mathrm{OH})^{80}$, Montroseit $(\mathrm{V}, \mathrm{Fe}) \mathrm{O}(\mathrm{OH})^{81},{ }^{82}$, Groutit $\alpha-\mathrm{MnO}(\mathrm{OH})^{83,84}$ und Goethit $\alpha-\mathrm{FeO}(\mathrm{OH})^{77}$. Kristallstrukturanalysen sind ferner durchgeführt worden von Böhmit $\gamma$ - $\mathrm{AlO}(\mathrm{OH})^{54,85-87}$ und den damit isostrukturellen Oxidhydroxiden $\gamma-\mathrm{ScO}(\mathrm{OH})^{85,88}$, $\gamma-\mathrm{CrO}(\mathrm{OH})^{89}$ und $\gamma-\mathrm{FeO}(\mathrm{OH})^{90}$; von $\gamma-\mathrm{BO}(\mathrm{OH})^{91}$, $\mathrm{Si}_{2} \mathrm{O}_{3}(\mathrm{OH})_{2} \mathrm{I}$ und $\mathrm{II}{ }^{92,93}$ und $\mathrm{As}_{3} \mathrm{O}_{5}(\mathrm{OH})_{5}{ }^{94,95}$; von den Mitgliedern der homologen Serie der allgemeinen Zusammensetzung $\mathrm{H}_{2 \mathrm{n}-2} \mathrm{~V}_{\mathrm{n}} \mathrm{O}_{3 \mathrm{n}-2}$ mit $n=4,6$ und $\infty$ : Häggit $\mathrm{V}_{2} \mathrm{O}_{3}(\mathrm{OH})_{3}{ }^{96-98}$, Doloresit $\mathrm{V}_{3} \mathrm{O}_{4}(\mathrm{OH})_{4}$ oder $\mathrm{V}_{3} \mathrm{O}_{3}(\mathrm{OH})_{5}{ }^{96-98}$ und Duttonit $\mathrm{VO}(\mathrm{OH})_{2}{ }^{97}, 99$; von den isostrukturellen Verbindungen $\mathrm{InOOH}{ }^{100}$ und $\mathrm{CrOOH}(\mathrm{Pnnm})^{101,102}$; von



Abb. 2. Kristallstruktur von Diaspor $\alpha-\mathrm{AlO}(\mathrm{OH})$. Projektion parallel [001]. Die stark und die schwach gezeichneten Atome befinden sich auf Spiegelebenen mit $z=\frac{1}{4}$ bzw. $z=-\frac{1}{4}$. Nach BusING und LEVY ${ }^{76}$.

den isostrukturellen Oxidhydroxiden $\alpha$ - $\mathrm{CrOOH}^{103-106}$ und $\mathrm{CoOOH}(\mathrm{R} \overline{3} \mathrm{~m})^{107}$; von $\gamma-\mathrm{MnO}(\mathrm{OH})^{108-110}$ und $\mathrm{U}_{2} \mathrm{O}_{5}(\mathrm{OH})_{2}{ }^{111}$, 112; von den isostrukturellen Verbindungen $\beta$ - $\mathrm{UO}_{2}(\mathrm{OH})_{2}{ }^{111-115}$ und $\mathrm{NpO}_{2}(\mathrm{OH})_{2}{ }^{116}$.

\section{$\gamma$ ) Hydroxy- und Oxysäuren}

Diskrete Moleküle der Art $\mathrm{M}(\mathrm{OH})_{\mathrm{m}}$ bzw. $\mathrm{M}(\mathrm{OH})_{m} \mathrm{O}_{\mathrm{n}}$ werden durch $\mathrm{O}-\mathrm{H} \cdots \mathrm{O}$-Bindungen zusammengehalten. Bei den Hydroxysäuren gehen von jedem $\mathrm{O}$-Atom zwei $\mathrm{O}-\mathrm{H} \cdots \mathrm{O}$-Bindungen aus. Die Struktur von $\mathrm{B}(\mathrm{OH})_{3}(\mathrm{PT} ; Z=4)$ ist aus $\mathrm{B}(\mathrm{OH})_{3}$-Molekülen aufgebaut, die durch fast lineare Wasserstoffbrücken [mittlerer $\mathrm{O}(\mathrm{H}) \mathrm{O}$-Abstand $2,720 \AA]$ miteinander verbunden sind und endlose Schichten nahezu hexagonaler Symmetrie bilden. Nach einer Neutronenbeugungs-Analyse an $\mathrm{D}_{3}{ }^{11} \mathrm{BO}_{3}$ betragen die mittleren Abstände $\mathrm{O}-\mathrm{D}$ 0,970 , $\mathrm{O}$ (D) $02,713 \AA, \mathrm{D} \cdots \mathrm{O} 1,744 \AA$ und der Bindungswinkel $\mathrm{O}-\mathrm{D} \cdots \mathrm{O} 177,9^{\circ}$. Die Struktur kann als Übereinanderlagerung solcher durch van der $\mathrm{W}$ a a ls scher Kräfte zusammengehaltener Schichten angesehen werden ${ }^{117-124}$ (Abb. 3) .

Bei den Oxysäuren sind die Sauerstoffatome teilweise an weniger als zwei $\mathrm{O}-\mathrm{H} \cdots \mathrm{O}$-Bindungen beteiligt $\left[\right.$ z. B. $\left.\mathrm{SO}_{2}(\mathrm{OH})_{2}{ }^{128-130}\right]$ und bei einem $\mathrm{H}: \mathrm{O}$-Verhältnis $<0,5$ geht höchstens eine $\mathrm{O}-\mathrm{H} \cdots \mathrm{O}$-Bindung von Sauerstoffatomen aus, die anderen Sauerstoffatome beteiligen sich nicht an dieser Bindung. Im $\alpha-\mathrm{IO}_{2}(\mathrm{OH})\left(\mathrm{P} 2_{1} 2_{1} 2_{1} ; Z=4\right)$ hat jedes Jodatom, zusätzlich zu seinen drei nächsten 


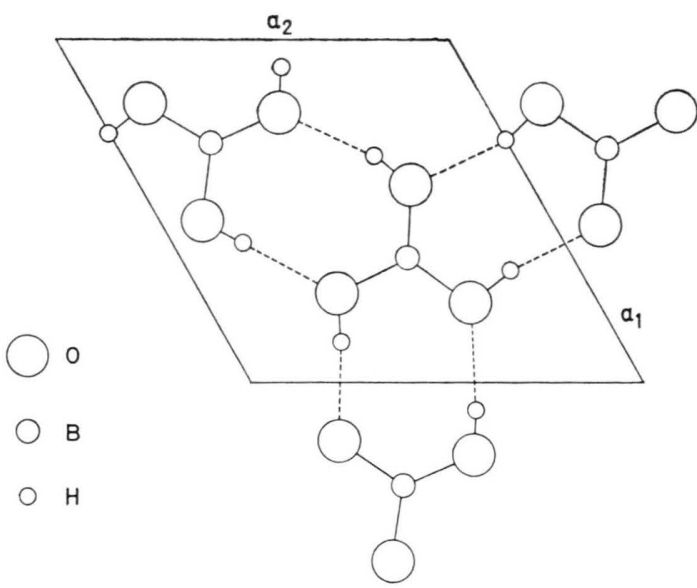

Abb. 3. Kristallstruktur von $\mathrm{B}(\mathrm{OH})_{3}$. Struktur einer Schicht. Nach ZACHARIASEN ${ }^{117}$.

O-Nachbarn in einer $\mathrm{IO}_{3}$-Gruppe, drei etwas weiter entfernte O-Nachbarn in Abständen, die wesentlich kleiner sind als die, welche man beim Vorliegen von van der Waals scher Bindung erwarten muß. Jod bildet somit drei schwächere und drei starke Bindungen zu Sauerstoff. Abgesehen von den kurzen $\mathrm{O}$-- O-Abständen, die Kanten der verzerrten $\mathrm{IO}_{6}$-Oktaeder sind, ist jede $\mathrm{IO}_{3}$-Gruppe mit seinen benachbarten Gruppen nur durch zwei lineare $\mathrm{O}-\mathrm{H} \cdots \mathrm{O}$-Bindungen $\left[\mathrm{O}_{\mathrm{II}}(\mathrm{H}) \ldots \mathrm{O}_{\mathrm{I}^{-}}\right.$Abstand $2,686 \AA ; \mathrm{O}_{\mathrm{II}} \ldots \mathrm{H}$ Abstand $0,99 \AA$ ] verbunden ${ }^{131-134}$. Ferner sind von den folgenden Hydroxy- bzw. Oxysäuren Kristallstrukturanalysen durchgeführt worden: $\mathrm{B}_{3} \mathrm{O}_{3}(\mathrm{OH})_{3}$ siehe ${ }^{135,136}, \mathrm{NO}_{2}(\mathrm{OH})^{137}, \mathrm{SeO}_{2}(\mathrm{OH})_{2}{ }^{138}, \mathrm{SeO}(\mathrm{OH})_{2}$ siehe ${ }^{139}, \mathrm{Te}(\mathrm{OH})_{6}$ kubisch $^{140}$, tetragonal ${ }^{140 \mathrm{a}}$ und monoklin ${ }^{140 a}$, $\mathrm{IO}(\mathrm{OH})_{5}{ }^{141-143}$ und $\mathrm{I}_{3} \mathrm{O}_{7}(\mathrm{OH})^{144}$.

Im Gegensatz zum strukturellen Aufbau der anderen Hydroxysäuren ist die Phosphorige Säure $\left(\mathrm{Pna}_{1} ; Z=8\right)$ aus $\mathrm{HPO}(\mathrm{OH})_{2}$-Molekülen aufgebaut. Das P-Atom und drei O-Atome bilden eine pyramidale Gruppe, die vierte tetraedrische Position ist wahrscheinlich von einem H-Atom besetzt. Die Lagen der Protonen konnten bei der Röntgenstrukturanalyse nicht festgelegt werden. Sie wurden aus kurzen intermolekularen $0 \ldots$...Abständen abgeleitet. Nach Infrarotspektren ${ }^{145}$ liegt eine $\mathrm{P}-\mathrm{H}$-Bindung vor. Die $\mathrm{HPO}(\mathrm{OH})_{2}$-Moleküle werden durch Wasserstoffbrücken (Länge 2,58-2,63 $\AA$ ) zusammengehalten ${ }^{146}$.

\section{Oxidhydrate}

Dies sind Verbindungen schwacher Säuren mit Wasser, die den Wasserstoff in Form von $\mathrm{H}_{2} \mathrm{O}$ -
Molekülen enthalten. Dabei können die Wassermoleküle in Molekül- oder Raumnetzstrukturen sowohl koordinativ an ein Metallatom gebunden sein oder auch als weitgehend isolierte starre oder rotierende Moleküle an festen Gitterplätzen vorliegen. Ein Beispiel für ein Oxidhydrat mit Molekülstruktur ist das Dirheniumdihydratoheptoxid. Im $\mathrm{Re}_{2} \mathrm{O}_{7}\left(\mathrm{OH}_{2}\right)_{2}\left(\mathrm{P} 2_{1} / \mathrm{m} ; \mathrm{Z}=4\right)$ ist ein Rheniumatom tetraedrisch, das andere verzerrt oktaedrisch von O-Atomen umgeben, wobei die tetraedrische Gruppe mit der oktaedrischen durch eine gemeinsame Ecke verknüpft ist. Da die Lage der Protonen röntgenographisch nicht bestimmbar war, mußte aus der verschiedenen Koordination der O-Atome auf die Art der Bindung des Wasserstoffs geschlossen werden. Pro Molekül haben nur zwei endständige O-Lagen einen um etwa $20 \%$ größeren Abstand vom oktaedrisch-koordinierten Re, so daß eine eindeutige Entscheidung zugunsten der Formulierung $\mathrm{Re}_{2} \mathrm{O}_{7}\left(\mathrm{OH}_{2}\right)_{2}$ möglich ist. Diese Moleküle sind nur durch Wasserstoffbrücken miteinander verbunden, deren Lage noch nicht eindeutig festgelegt werden kann ${ }^{14}$ (Abb. 4).



Abb. 4. Kristallstruktur von $\mathrm{Re}_{2} \mathrm{O}_{7} \cdot 2 \mathrm{H}_{2} \mathrm{O}$. Projektion parallel [010]. Genau übereinanderliegende Atome wurden etwas versetzt. Nach Beyer, Glemser und Krebs ${ }^{147}$.

Die als „Molybdän-“ und „Wolframsäuren“ bezeichneten Substanzen enthalten keine diskreten $\mathrm{H}_{2} \mathrm{MO}_{4}$-Moleküle. Diese Verbindungen sind Oxidhydrate: $\mathrm{MoO}_{3} \cdot 2 \mathrm{H}_{2} \mathrm{O}, \quad \mathrm{MoO}_{3} \cdot \mathrm{H}_{2} \mathrm{O}, \quad \mathrm{WO}_{3} \cdot 2 \mathrm{H}_{2} \mathrm{O}$ und $\mathrm{WO}_{3} \cdot \mathrm{H}_{2} \mathrm{O} .{ }^{1} \mathrm{H}$-Breitlinien-NMR-Untersuchungen und IR-Spektren zeigen, daß der Wasserstoff in Form von Wassermolekülen vorhanden ist ${ }^{148-151}$. Die Molybdän-Verbindungen sind mit den WolframVerbindungen isotyp. Eine nicht vollständige Röntgenstrukturanalyse von $\mathrm{MoO}_{3} \cdot 2 \mathrm{H}_{2} \mathrm{O}(\mathrm{P} 2 / \mathrm{m} ; Z=2)$ zeigt die Anwesenheit von Schichten aus $\mathrm{MoO}_{6}$. Oktaedern, die gemeinsame Ecken haben ${ }^{152,153}$. 


\section{Hydratisierte Oxysäuren}

In diesen Verbindungen starker Säuren mit Wasser sind Protonen mit $\mathrm{H}_{2} \mathrm{O}-$ Molekülen verbunden unter Bildung von $\mathrm{H}_{3} \mathrm{O}^{\oplus}$-, $\mathrm{H}_{5} \mathrm{O}_{2}{ }^{\oplus}$ - und vermutlich noch höher hydratisierten Ionen (z. B. $\mathrm{H}_{7} \mathrm{O}_{3}{ }^{\oplus}$, $\left.\mathrm{H}_{9} \mathrm{O}_{4}{ }^{\oplus}\right)$. $\mathrm{H}_{3} \mathrm{O}^{\oplus}$-Ionen liegen im Kristallgitter von „Perchlorsäure-Monohydrat“, $\left(\mathrm{H}_{3} \mathrm{O}\right)^{\oplus}\left(\mathrm{ClO}_{4}\right)^{\ominus}$, vor. Die unterhalb $-30{ }^{\circ} \mathrm{C}$ stabile monokline Phase $(\mathrm{P} 21 / \mathrm{n} ; Z=4)$ besteht aus Schichten von tetraedrisch aufgebauten $\mathrm{ClO}_{4}{ }^{\ominus}$ - und pyramidal aufgebauten $\mathrm{H}_{3} \mathrm{O}^{\odot}$-Ionen, die durch Wasserstoffbrücken miteinander verbunden sind. In der oberhalb $-30{ }^{\circ} \mathrm{C}$ orthorhombischen Phase (Pnma; $Z=4$ ) liegt eine freie Rotation der $\mathrm{H}_{3} \mathrm{O}^{\oplus}$-Ionen vor ${ }^{154-157}$ (Abb. 5).

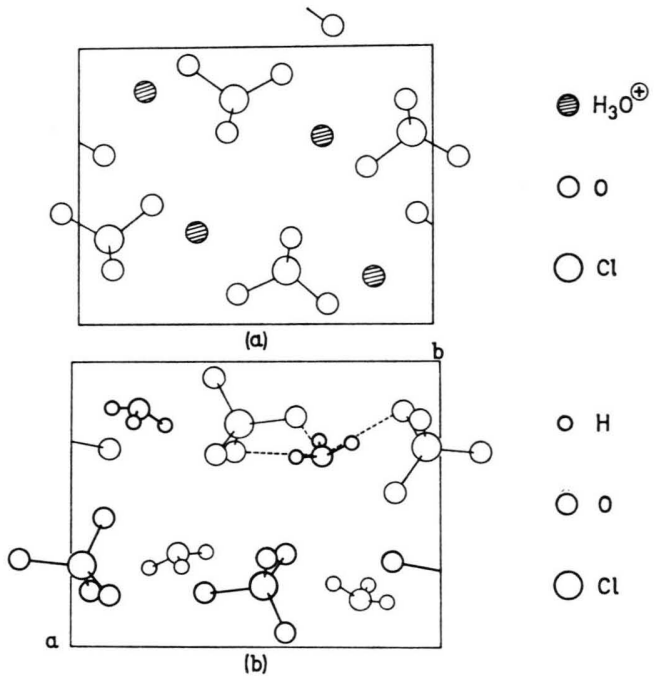

Abb. 5. Kristallstruktur von $\left(\mathrm{H}_{3} \mathrm{O}\right) \oplus\left(\mathrm{ClO}_{4}\right)^{\ominus}$. a) Schematische Projektion der Raumtemperatur-Struktur. Nach LEE und CARPENTER ${ }^{154}$. b) Entsprechende Projektion der Tieftemperatur-Struktur (-... Wasserstoffbrücken). Nach NORDMANN ${ }^{155}$.

Oxonium-Ionen liegen auch im „SalpetersäureMonohydrat “, $\left(\mathrm{H}_{3} \mathrm{O}\right)^{\oplus}\left(\mathrm{NO}_{3}\right)^{\ominus}$, vor ${ }^{158-160}$. Eine Verbindung der Bruttozusammensetzung $11 \mathrm{Al}_{2} \mathrm{O}_{3} \cdot 3 \mathrm{H}_{2} \mathrm{O}$ mit $\beta$ - $\mathrm{Al}_{2} \mathrm{O}_{3}$-Struktur wird als $\left(\mathrm{H}_{3} \mathrm{O}\right)_{2}\left(\mathrm{Al}_{22} \mathrm{O}_{34}\right)$ formuliert ${ }^{161}$. $\left(\mathrm{H}_{5} \mathrm{O}_{2}\right)^{\oplus}$-Ionen treten im „PerchlorsäureDihydrat"“, $\left(\mathrm{H}_{5} \mathrm{O}_{2}\right)^{\oplus}\left(\mathrm{ClO}_{4}\right)^{\ominus}$, auf. In dieser Verbindung werden die Wassermoleküle paarweise durch eine sehr kurze Wasserstoffbrücke $(2,424 \AA)$ zusammengehalten. Auf Grund der gewählten Raumgruppe (Pnma; $Z=4$ ) befindet sich auf der Mitte dieser Brücke ein Symmetriezentrum. Die Kürze der Brücke wie auch die symmetrische Umgebung des Wasserstoffatoms in der Mitte führen zu der Annahme einer sehr flachen, möglicherweise symmetri- schen Potentialenergiekurve für die Bewegung des Protons oder eines Doppelminimum-Potentials mit einer niedrigen Potentialbarriere. Die $\left(\mathrm{H}_{5} \mathrm{O}_{2}\right)^{\oplus}$. Ionen sind durch Wasserstoffbrücken mit $\left(\mathrm{ClO}_{4}\right)^{\ominus}$. Ionen verbunden. Es bilden sich so senkrecht zur $a$-Achse Schichten aus, die durch van der W a a ls sche Kräfte zusammengehalten werden ${ }^{162}$ (Abb.6) .

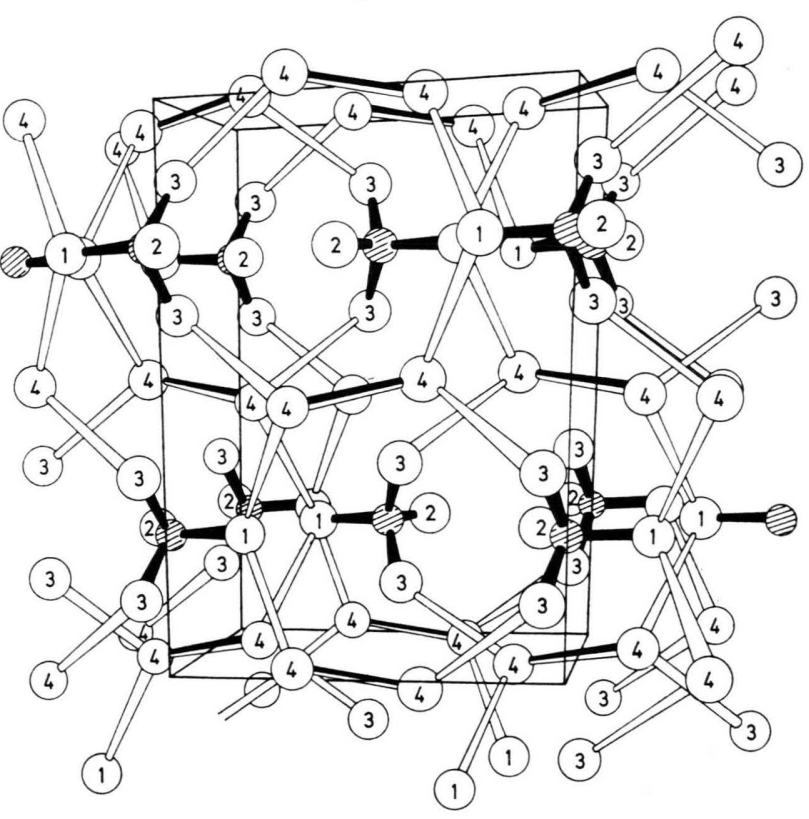

(a)

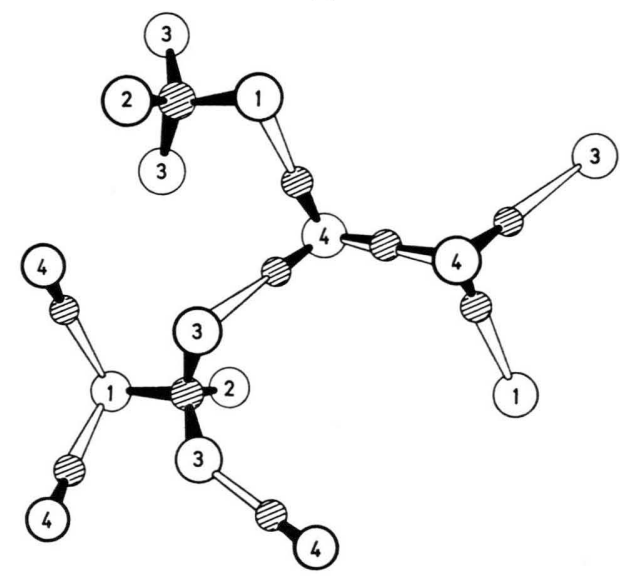

(b)

Abb. 6. Kristallstruktur von $\left(\mathrm{H}_{5} \mathrm{O}_{2}\right) \oplus\left(\mathrm{ClO}_{4}\right)^{\ominus}$. a) Stereoskopische Zeichnung. Kovalente Bindungen im $(\mathrm{ClO})_{4}{ }^{\ominus}$.Ion gefüllt; Wasserstoffbrückenbindungen leer mit Ausnahme der $\mathrm{O}(4) \ldots \mathrm{O}(4)$-Bindungen, die halbgefüllt sind. O-Atome numeriert wie in $b$, Wasserstoffatome nicht erfaßt. b) Anordnung der H-Atome: $1,0 \AA$ von $O(4)$ entfernt in der $\mathrm{O}(4) \ldots \mathrm{O}(1)$ - und $\mathrm{O}(4) \ldots \mathrm{O}(3)$-Wasserstoffbrücke und auf der Mitte der $\mathrm{O}(4) \ldots \mathrm{O}(4)$-Bindung. Nach OLOvsson ${ }^{162}$. 
Berechnungen über die Stabilität von $\mathrm{H}_{5} \mathrm{O}_{2}{ }^{\oplus}$ relativ zu $\mathrm{H}_{3} \mathrm{O}^{\oplus}$ und $\mathrm{H}_{2} \mathrm{O}$ sind durchgeführt worden ${ }^{163}$.

Unterschiedliche Bindungsverhältnisse für den Wasserstoff liegen in den folgenden Verbindungen der Systeme Oxid-Wasser vor: $\mathrm{Li}(\mathrm{OH}) \cdot \mathrm{H}_{2} \mathrm{O}^{164-166}$, $\mathrm{Na}(\mathrm{OH}) \cdot \mathrm{H}_{2} \mathrm{O}^{167}, 2 \mathrm{Na}(\mathrm{OH}) \cdot 7 \mathrm{H}_{2} \mathrm{O}^{167}, \mathrm{~K}(\mathrm{OH}) \cdot$ $\mathrm{H}_{2} \mathrm{O}^{168}, \mathrm{Sr}(\mathrm{OH})_{2} \cdot \mathrm{H}_{2} \mathrm{O}^{169}$ und die damit isotypen Verbindungen $\mathrm{Ba}(\mathrm{OH})_{2} \cdot \mathrm{H}_{2} \mathrm{O}^{169}$ und $\mathrm{Eu}(\mathrm{OH})_{2} \cdot$ $\mathrm{H}_{2} \mathrm{O}^{169}, \mathrm{Sr}(\mathrm{OH})_{2} \cdot 8 \mathrm{H}_{2} \mathrm{O}^{170,171}, \mathrm{Ba}(\mathrm{OH})_{2} \cdot 8 \mathrm{H}_{2} \mathrm{O}^{172}$, $\mathrm{B}_{3} \mathrm{O}_{4}(\mathrm{OH})\left(\mathrm{OH}_{2}\right)=, \beta-\mathrm{HBO}_{2}{ }^{\prime 173}, 2 \mathrm{PO}(\mathrm{OH})_{3} \cdot \mathrm{H}_{2} \mathrm{O}$ siehe ${ }^{173 a}$ und das damit isomorphe $2 \mathrm{AsO}(\mathrm{OH})_{3}$. $\mathrm{H}_{2} \mathrm{O}^{174},\left(\mathrm{H}_{3} \mathrm{O}\right)\left[\mathrm{SeO}_{3}(\mathrm{OH})\right]^{175},\left(\mathrm{H}_{3} \mathrm{O}\right)\left[\mathrm{SO}_{3}(\mathrm{OH})\right]$ s. ${ }^{160,176}, \mathrm{U}_{2} \mathrm{O}_{5}(\mathrm{OH})_{2} \cdot 3 \mathrm{H}_{2} \mathrm{O}^{112,177}$ und $\mathrm{Np}_{2} \mathrm{O}_{5}(\mathrm{OH})_{2}$. $3 \mathrm{H}_{2} \mathrm{O}^{116}$.

4. Feste Lösung von Protonen in e in em Oxidgitter: $\mathrm{Bronzen} \mathrm{H}_{\mathrm{x}} \mathrm{WO}_{3}$

Die Wolframbronzen sind nichtstöchiometrische Substanzen, im Falle der Wasserstoffverbindungen mit der Formel $\mathrm{H}_{\mathrm{x}} \mathrm{WO}_{3}(x=0,1 ; 0,23 ; 0,33 ; 0,5)$. Strukturell können diese Verbindungen als Wasserstoff-Unterschuß-HWO $\mathrm{H}_{3}$-Phasen mit gestörter Perowskit-Struktur angesehen werden. Die Kristallstruktur besteht aus deformierten $\mathrm{WO}_{6}$-Oktaedern, die über Ecken miteinander verbunden sind. Die Wasserstoffatome sind statistisch verteilt und besetzen einen Teil der größeren Zwischengitterplätze des Kristallgitters (Abb. 7). Bei $\mathrm{H}_{0.25} \mathrm{WO}_{3}$ betragen die $\mathrm{H} \ldots \mathrm{O}-\mathrm{Ab}$ stände $2,62 \AA$ und $2,68 \AA$, sie sind deutlich länger als beim Vorliegen einer $\mathrm{O}-\mathrm{H}$-Bindung. Der O...O-Abstand beläuft sich auf etwa $5 \AA$ und kommt für eine Wasserstoffbrückenbindung nicht in Frage. Der W...H-Abstand ist 3,10 $\AA$, beträchtlich größer als Metall - H-Abstände in Hydridkomplexen und viel zu groß für eine $\mathrm{W}-\mathrm{H}$-Bindung. Darüberhinaus sind die W $5 \mathrm{dt}_{2 \mathrm{~g}}$-Orbitale so orientiert, daß sie keine ausgedehnte Überlappung mit dem Wasserstofforbital ergeben. Am besten kann man die Wolframbronzen $\mathrm{H}_{\mathrm{X}} \mathrm{WO}_{3}$ als eine feste Lösung von Protonen in $\mathrm{WO}_{3}$ beschreiben. Die Struktur von $\mathrm{H}_{0,23} \mathrm{WO}_{3} \quad(\mathrm{P} 4 / \mathrm{nmm} ; Z=2)$ ist identisch mit der von $\mathrm{Na}_{0,1} \mathrm{WO}_{3}{ }^{178-181}$.

Auch von $\mathrm{MoO}_{3}$ leiten sich genotypische Verbindungen mit der Bruttoformel $\mathrm{H}_{\mathrm{x}} \mathrm{MoO}_{3} \quad(x=0,5$; $1,0 ; 1,6 ; 2,0)$ ab ${ }^{182-185}$. Die Struktur von $\mathrm{H}_{0.5} \mathrm{MoO}_{3}(\mathrm{Cmcm} ; Z=4)$ ähnelt sehr der von $\mathrm{MoO}_{3}$ (Pbnm: $Z=4)^{186}$. Die Art der Bindung des Wasserstoffs ist noch nicht bekannt.

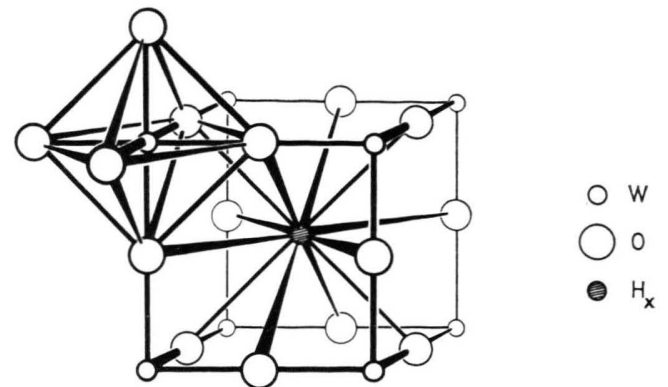

Abb. 7. Perowskitstruktur der Wasserstoffanaloga von Wolframbronzen $\mathrm{H}_{\mathrm{X}} \mathrm{WO}_{3}$.

\section{Festlegung des Bindungstyps}

Auf welche Weise läßt sich die Bindung des Wasserstoffs in kristallinen Aquoxiden festlegen? Zur Lösung dieser Aufgabe dürfen nur solche Methoden herangezogen werden, die den strukturellen Aufbau des Präparats während der Untersuchung unverändert lassen. Chemische Reaktionen sind deshalb meistens nicht geeignet. Eine Entscheidung über das Vorliegen eines bestimmten Bindungstyps ist mit Sicherheit dann zu treffen, wenn die vollständige Kristallstruktur der Verbindung bekannt ist. Durch Röntgen-, Neutronen- und Elektronenbeugungs-Analysen lassen sich alle Atomlagen prinzipiell festlegen. Jedoch ist es bei den hier häufig polykristallin vorliegenden Substanzen äußerst schwierig, die Lagen der H-Atome zu bestimmen. Eine wesentlich schnellere Information über die Bindung des Wasserstoffs ist durch Messung des IR-Spektrums, gegebenenfalls unter Heranziehen des $\mathrm{R}$ aman -, INS- und ${ }^{1} \mathrm{H}$ NMR-Breitlinienspektrums, möglich, und zwar sowohl an Einkristallen wie auch an polykristallinen Substanzen und ohne genaue Kenntnis der Kristallstruktur.

\section{IR-Spektren von Hydroxiden}

Im IR-Spektrum von Hydroxiden treten neben anderen auch solche Schwingungsbanden auf, die vielfach als hauptsächlich von den Schwingungen der $\mathrm{OH}$-Gruppe herrührend angesehen werden können. Ein $\mathrm{OH}^{\ominus}$-Ion kann im Kristallgitter $\mathrm{OH}^{\ominus}$-Valenz$\left({ }^{\nu} \mathrm{OH}^{\ominus}\right)$ und $\mathrm{OH}^{\ominus}$-Librationsschwingungen $\left({ }^{\delta} \mathrm{OH}^{\ominus}\right)$ ausführen. Liegt Wasserstoffbrückenbindung der Art $\mathrm{O}-\mathrm{H} \cdots \mathrm{O}$ vor, dann hat man es mit OH-Valenz- $\left({ }^{\nu} \mathrm{OH}\right)$ oder $\mathrm{OH}$-Librationsschwingungen $\left({ }^{\delta} \mathrm{OH}\right)$ zu tun. Letztere werden bei stärkerer Wechselwirkung der OH-Gruppe mit dem assoziierten O-Atom 
auch OH-Deformationsschwingungen genannt. Die Wellenzahl der $\mathrm{OH}^{\ominus}$.Valenzschwingung liegt ungefähr im Bereich von 3700 bis $3600 \mathrm{~cm}^{-1}$, die der $\mathrm{OH}^{\ominus}$-Librationsschwingung zwischen 300 und $500 \mathrm{~cm}^{-1}$. Je nach der Stärke der Wasserstoffbrükkenbindung liegt das Maximum der Absorption von der $\mathrm{OH}-$ Valenzschwingung im Bereich von etwa 3600 bis $1600 \mathrm{~cm}^{-1}$, das der OH-Librations- bzw. OH-Deformationsschwingung ungefähr zwischen 500 und $1200 \mathrm{~cm}^{-1} 187-194$.

\section{IR-Spektren von Oxidhydraten}

Im IR-Spektrum von Oxidhydraten treten im Prinzip dieselben Schwingungsbanden wie im freien Wassermolekül auf. Das freie $\mathrm{H}_{2} \mathrm{O}$-Molekül zeigt 3 Grundschwingungen und zwar je eine symmetrische $\left(v_{1, \mathrm{HOH}}\right)$ und asymmetrische $\left(v_{3, \mathrm{HOH}}\right) \mathrm{HOH}$ Valenzschwingung bei $3652 \mathrm{bzw} .3756 \mathrm{~cm}^{-1}$, sowie eine $\mathrm{HOH}$-Deformationsschwingung $\left(v_{2, \mathrm{HOH}}\right)$ bei $1595 \mathrm{~cm}^{-1}$. Im kristallinen Zustand sind je nach der Stärke der Wasserstoffbrückenbindung und sonstiger Wechselwirkungen der HOH-Moleküle mit dem Rest des Kristallgitters die $\mathrm{HOH}-V$ alenzschwingungen im Bereich von etwa 3500 bis $3000 \mathrm{~cm}^{-1}$, die HOH-Deformationsschwingungen zwischen 1550 und $1650 \mathrm{~cm}^{-1}$ zu erwarten. Im ferneren IR-Bereich treten daneben weitere Banden auf, die von einer gehinderten Rotation und einer Translation der HOH-Moleküle herrühren.

\section{IR-Spektren von hydratisierten Oxysäuren}

Im IR-Spektrum von Oxonium-Verbindungen treten bei hinreichend tiefen Temperaturen, wenn die Rotation der $\mathrm{H}_{3} \mathrm{O}^{\oplus}$-Ionen zum Stillstand gekommen ist, Banden von vier Grundschwingungen auf, die für das pyramidal aufgebaute $\mathrm{H}_{3} \mathrm{O}^{\oplus}$-Ion charakteristisch sind, neben Librations- und Translationsschwingungsbanden. In den bisher untersuchten Oxonium-Verbindungen wurden die einigermaßen lagekonstanten Banden wie folgt zugeordnet: Die Absorption im Bereich von 3400 bis $3100 \mathrm{~cm}^{-1}$ ist eine Bande von der antisymmetrischen Valenzschwingung, diejenige zwischen 2650 und $2450 \mathrm{~cm}^{-1}$ eine Bande von der symmetrischen Valenzschwingung. Die Absorption um $1700 \mathrm{~cm}^{-1}$ rührt von der antisymmetrischen Deformationsschwingung, die um $1200 \mathrm{~cm}^{-1}$ von der symmetrischen Deformationsschwingung her. Letztere ist bisweilen durch Wechselwirkung im Kristallgitter aufgespalten. Die Wel- lenzahlen der Librationsschwingungen liegen bei etwa $700 \mathrm{~cm}^{-1}$, die der Translationschwingungen zwischen 120 und $400 \mathrm{~cm}^{-1194 a}$.

Im IR-Spektrum von $\mathrm{H}_{5} \mathrm{O}_{2}{ }^{\oplus}$-Verbindungen wurden Banden um 2900, 2230, 1675 und $970 \mathrm{~cm}^{-1}$ beobachtet ${ }^{195,196}$. Ein typisches Spektrum von $\mathrm{H}_{5} \mathrm{O}_{2}{ }^{\oplus}$ findet sich in Abb. 8. Zum Vergleich sind typische Spektren von $\mathrm{H}_{3} \mathrm{O}^{\odot}$ und Kristallwasser angegeben.



Abb. 8. Typische IR-Spektren von $\mathrm{H}_{2} \mathrm{O}, \mathrm{H}_{3} \mathrm{O}^{\oplus}$ und $\mathrm{H}_{5} \mathrm{O}_{2}{ }^{\oplus}$.

\section{IR-Spektren von Bronzen $\mathrm{H}_{x} \mathrm{WO}_{3}$}

In den IR-Spektren der Wasserstoffanaloga von Wolframbronzen $\mathrm{H}_{\mathrm{x}} \mathrm{WO}_{3}$ tritt im untersuchten Bereich von 4000 bis $200 \mathrm{~cm}^{-1}$ neben den Banden von $\mathrm{WO}_{3}$ nur eine ziemlich scharfe Absorption bei $690 \mathrm{~cm}^{-1}$ auf. Diese Bande verliert an Intensität, wenn sich die Substanzen an der Luft allmählich oxydieren und verschwindet beim Glühen von $\mathrm{H}_{\mathrm{x}} \mathrm{WO}_{3}$ an der Luft vollständig. Sehr wahrscheinlich ist sie einer Bewegung der H-Atome in der [001]-Richtung in das angenähert ebene Quadrat von O-Atomen, die bei $\mathrm{H}_{0,25} \mathrm{WO}_{3}$ durch die speziellen Lagen (c) der Raumgruppe P4/nmm gegeben sind, hinein und heraus zuzuordnen. Eine solche Bewegung würde einer Trampolin-Bewegung ähneln, die - wegen der großen Atomabstände - vermutlich eine geringe Kraftkonstante besäße und von ziemlich geringer Frequenz sein würde ${ }^{197}$.

\section{Verlauf der potentiellen Energie des Protons in einer $\mathrm{O}-\mathrm{H} \cdots \mathrm{O}$-Brücke}

In der Literatur liegen ziemlich umfangreiche Berechnungen über die Bindung in $\mathrm{O}-\mathrm{H} \cdots \mathrm{O}$-Brükken vor ${ }^{198}$, 199 . Jedoch ist eine Beziehung zwischen der potentiellen Energie des Protons in der 
$\mathrm{O}-\mathrm{H} \cdots \mathrm{O}$-Brücke und dem $\mathrm{O}(\mathrm{H}) \ldots \mathrm{O}$-Abstand noch nicht bekannt. Nur in einigen Fällen kann man aus den experimentellen Befunden auf den Charakter dieser Funktion schließen. Prinzipiell kann man für die Valenzschwingung des Protons in der Wasserstoffbrücke verschiedene Formen von Kurven der potentiellen Energie betrachten (Abb. 9). Bei schwacher Wasserstoffbrückenbindung, z. B. bei $\mathrm{K}(\mathrm{OH})[\mathrm{O}(\mathrm{H}) \ldots \mathrm{O}$-Abstand $3,35 \AA]$, kann für die Bewegung des Protons, das sich ungefähr auf der $\mathrm{O}_{\mathrm{II}} \ldots \mathrm{O}_{\mathrm{I}^{-}}$Verbindungslinie nur in der Nähe des $\mathrm{O}_{\mathrm{II}}$-Atoms aufhält, eine asymmetrische Kurve der potentiellen Energie mit nur einem Minimum etwa $1 \AA$ von $\mathrm{O}_{\mathrm{II}}$ entfernt angenommen werden (Kurve a). Beim anderen Extrem, z. B. bei $\mathrm{H}_{5} \mathrm{O}_{2}{ }^{\oplus}[\mathrm{O}(\mathrm{H}) \mathrm{O}$ Abstand 2,4-2,5 $\left.\AA^{200}\right]$ und $\alpha-\mathrm{CrOOH}[\mathrm{O}(\mathrm{H}) \mathrm{O}$. Abstand 2,49 $\AA$, liegt dann eine symmetrische Kurve der potentiellen Energie mit einem Minimum in der Mitte der Wasserstoffbrücke vor, da das Proton gleichweit von den O-Atomen $\mathrm{O}_{\mathrm{I}}$ und $\mathrm{O}_{\text {II }}$ entfernt ist (Kurve $\mathrm{f}$ ). In den Fällen dazwischen, also bei Verbindungen mit mittelstarker Wasserstoffbrückenbindung, haben die Kurven der potentiellen Energie zwei Minima von gleicher (Kurve b und c)

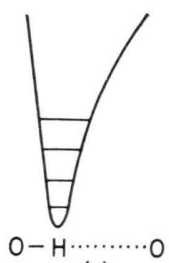

(a)

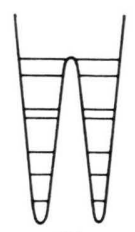

(b)

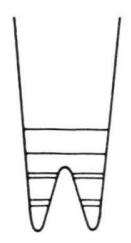

(c)

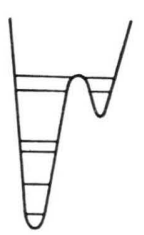

(d)

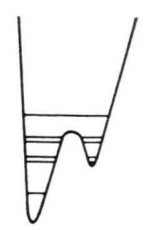

(e)



(f)
Abb. 9. Angenommener Verlauf der potentiellen Energie des Protons in einer $\mathrm{O}-\mathrm{H}$... O-Brücke. Von a bis $\mathrm{f}$ mit abnehmendem $\mathrm{O}(\mathrm{H}) \mathrm{O}$-Abstand. und unterschiedlicher (Kurven d und e) Tiefe. Während die Deutung der OH-Valenzschwingungsbanden in den IR-Spektren von Verbindungen mit schwacher Wasserstoffbrückenbindung ohne weiteres möglich ist, tritt bei starker Wasserstoffbrückenbindung ein Phänomen auf, das bei schwacher Wasserstoffbrückenbindung kaum eine Rolle spielen dürfte und das die Zuordnung von Banden zu OH-Valenzschwingungen erheblich erschwert. Es ist dies der Tunnelproze $ß$ des Protons in einem Potential mit Doppelminimum und als Folge davon eine Aufspaltung der Schwingungsniveaus. Nach Berechnungen von REID ${ }^{201}$ sollte ein solches Doppelminimum-Potential für Wasserstoffbrückenbindungen oberhalb eines $\mathrm{O}(\mathrm{H}) \ldots \mathrm{O}$-Abstandes von $2,52 \AA$ vorliegen, unterhalb dieses Abstandes sollten beide Minima in ein einziges zusammenlaufen. Doch sind die Theorien und die Berechnungen der Energieniveaus normalerweise nur für einfache Modelle entwickelt worden - alle drei Atome in der $\mathrm{O}-\mathrm{H}-\mathrm{O}$-Brücke schwingen in einer Dimension -, wohingegen die tatsächlich vorliegenden Systeme viel mehr Freiheitsgrade und verschiedene Arten von Wechselwirkungen besitzen, so daß ein Vergleich von wirklich gemessenen und theoretisch vorhergesagten Spektren schwierig ist. Nichtsdestoweniger dürfte dieser Effekt einen Beitrag zur detaillierten Bandenform einschließlich einer geringen Aufspaltung der Banden liefern.

\section{Spezielle Aussagen über die Bindung des Wasserstoffs in Hydroxiden}

Auf Grund der für die einzelnen Bewegungen des Protons charakteristischen Schwingungsbanden im IR-Spektrum kann man nun die Art der Bindung des Wasserstoffs prinzipiell festlegen. Nehmen wir als Beispiel den Hydrargillit, eine Verbindung, die sich formal aus einer Formeleinheit Aluminiumoxid und drei Formeleinheiten Wasser ableitet. Im IRSpektrum treten - neben $\mathrm{Al}$ - O-Schwingungsbanden - acht Absorptionsbanden auf, die auf Grund ihrer Frequenzlage und ihrem Verhalten beim Deuterieren hauptsächlich auf $\mathrm{OH}-\mathrm{Valenz}$ - und $\mathrm{OH}$-Deformationsschwingungen zurückzuführen sind. Hydrargillit muß demnach als Hydroxid formuliert werden mit der Formel $\mathrm{Al}(\mathrm{OH})_{3}$. Es erhebt sich aber nun die Frage, wie das Auftreten so zahlreicher OH-Banden im IR-Spektrum zu deuten ist. Welche weiterreichenden Aussagen sind darin eventuell noch 
enthalten? Betrachten wir dazu die Struktur des monoklinen Hydrargillits. Dieses Hydroxid kristallisiert in der Raumgruppe $\mathrm{P} 2 / \mathrm{n}$. Es befinden sich 8 Formeleinheiten in der Einheitszelle. Diese Zelle enthält somit 24 OH-Gruppen, je 2 OH-Gruppen sind durch ein Symmetriezentrum miteinander verbunden. Im IR-Spektrum sind Banden von maximal $12 \mathrm{OH}-\mathrm{Valenz}$ - und 24 OH-Deformationsschwingungen zu erwarten, die asymmetrisch zum Symmetriezentrum erfolgen. Man kann annehmen, daß diese $\mathrm{OH}-B a n d e n$ über einen ziemlich weiten Wellenzahlbereich verstreut sein werden, da drei verschiedene kurze 0 . . O-Obstände $(2,80 \AA ; 2,84 \AA ; 2,87 \AA)^{59}$ im Kristallgitter auftreten, die wohl als Wasserstoffbrücken zu deuten sind. Die Protonenlagen selbst sind im Kristallgitter des Hydrargillits noch nicht bekann. Im IR-Spektrum von polykristallinem Material sind aber nur 4 OH-Valenz- (3617, 3520, 3428 und $3380 \mathrm{~cm}^{-1}$ ) und $4 \mathrm{OH}$-Deformationsschwingungsbanden $\left(1020,958,914\right.$ und $\left.743 \mathrm{~cm}^{-1}\right)$ erkennbar. Eine Erklärung dafür, wie diese Banden im einzelnen zu deuten sind, kann - solange die Protonenlagen unbekannt sind und Messungen im polarisierten IR-Strahl an orientierten gut ausgebildeten Einkristallen von Hydrargillit fehlen - noch nicht gegeben werden.

An strukturell sehr einfach gebauten Hydroxiden soll nun aber gezeigt werden, wie es überhaupt zu einer so großen Anzahl von OH-Banden kommen kann, einer großen Anzahl, wie sie in den IR-Spektren von Aquoxiden immer wieder beobachtet wird. Dazu werden IR-Absorptionsspektren von polykristallinen Hydroxiden angeführt, bei denen die $\mathrm{OH}$ Gruppen im Kristallgitter alle die gleiche Umgebung haben und die -- sofern die $\mathrm{OH}$-Gruppen isoliert voneinander betrachtet werden - auch nur eine $\mathrm{OH}$ Valenzschwingung und zwei OH-Librations- bzw. Deformationsschwingungen zeigen sollten.

\section{$\mathrm{Li}(\mathrm{OH})$}

Lithiumhydroxid kristallisiert in der Raumgruppe $\mathrm{P} 4 / \mathrm{nmm}$. Es befinden sich 2 Formeleinheiten in der Einheitszelle. Die beiden $\mathrm{OH}^{\ominus}$-Ionen sind durch ein Symmetriezentrum miteinander verbunden ${ }^{23}$. Im Kristallgitter liegt keine Wasserstoffbrükkenbindung vor ${ }^{24}$. Die Struktur besteht also aus $\mathrm{Li}^{\oplus}$ - und $\mathrm{OH}^{\ominus}$-Ionen. Im Schwingungsspektrum sind somit Banden von maximal $2 \mathrm{OH}^{\ominus}$.Valenzschwingungen und $4 \mathrm{OH}^{\ominus}$-Librationsschwingungen zu erwarten. Dabei darf nur die Hälfte dieser
Schwingungen infrarotaktiv sein und zwar nur diejenigen Schwingungen, die asymmetrisch zum Symmetriezentrum erfolgen. Im IR-Spektrum ${ }^{202-212}$ (Abb. 10) tritt eine scharfe Bande bei $3678 \mathrm{~cm}^{-1}$ auf, die einer $\mathrm{OH}^{\ominus}$-Valenzschwingung $\left(v_{\mathrm{OH}^{-}}\right)$zugeordnet wird ${ }^{202}$. Im langwelligen IR-Bereich erscheinen bei $\mathrm{Li}(\mathrm{OH})$ weitere Banden. Beim Deuterieren verschiebt sich nur die Bande bei $419 \mathrm{~cm}^{-1}$, sie tritt im IR-Spektrum von $\mathrm{Li}(\mathrm{OD})$ gesetzmäßig wegen des Massenunterschieds von Wasserstoff und Deuterium nach kleineren Wellenzahlen verschoben bei $304 \mathrm{~cm}^{-1}$ auf. Sie wird $\mathrm{OH}^{\ominus}$-Librationsschwingungen zugeordnet $\left(\delta_{\mathrm{OH}^{-}}\right)$, während die andere breite Absorption auf Translationsschwingungen des Gitters zurückgeführt wird ${ }^{203}$. Scharfe Banden von $\mathrm{OH}^{\ominus}$.Valenzschwingungen wurden auch beobachtet in den IR-Spektren von $\alpha-\mathrm{Na}(\mathrm{OH})\left[3637 \mathrm{~cm}^{-129}\right.$, 204, 213-215], $\mathrm{Mg}(\mathrm{OH})_{2} \quad\left[3649 \mathrm{~cm}^{-1}\right.$ 205, 215-226 $]$, $\mathrm{Ca}(\mathrm{OH})_{2}\left[3644 \mathrm{~cm}^{-1} 214,215,221-228\right], \mathrm{Mn}(\mathrm{OH})_{2}$ $\left[3655 \mathrm{~cm}^{-1}{ }^{189}\right], \quad \mathrm{Fe}(\mathrm{OH})_{2} \quad\left[\begin{array}{lll}3640 & \left.\mathrm{~cm}^{-1}{ }^{189}\right],\end{array}\right.$ $\mathrm{Co}(\mathrm{OH})_{2} \quad\left[\begin{array}{llll}3660 & \mathrm{~cm}^{-1} & 189\end{array}\right], \quad \mathrm{Ni}(\mathrm{OH})_{2} \quad\left[\begin{array}{lll}3655 & \end{array}\right.$ $\left.\mathrm{cm}^{-1}{ }^{189}\right], \quad \mathrm{Y}(\mathrm{OH})_{3} \quad$ [ca. $\left.3600 \mathrm{~cm}^{-1}{ }^{37},{ }^{229}\right]$, $\mathrm{La}(\mathrm{OH})_{3}\left[3645 \mathrm{~cm}^{-1} 229,230\right]$ und den Trihydroxiden der Seltenen Erdmetalle [3600-3645 $\left.\mathrm{cm}^{-1}{ }^{229}\right]$. Banden von $\mathrm{OD}^{\ominus}$-Librationsschwingungen wurden bei $\mathrm{Mg}(\mathrm{OD})_{2} \quad\left[310 \pm 10 \mathrm{~cm}^{-1}{ }^{222}\right]$ und $\mathrm{Ca}(\mathrm{OD})_{2}\left[265 \pm 30 \mathrm{~cm}^{-1}{ }^{222}\right]$ gefunden.

\section{$\mathrm{K}(\mathrm{OH})$}

Im Gegensatz zu $\mathrm{Li}(\mathrm{OH})$ tritt im Kristallgitter von $\mathrm{K}(\mathrm{OH})$ eine, wenn auch nur sehr schwache, Wasserstoffbrückenbindung zwischen den $\mathrm{OH}$-Gruppen auf. $\mathrm{K}(\mathrm{OH})$ kristallisiert in der Raumgruppe $\mathrm{P} 2{ }_{1}$ mit 2 Formeleinheiten in der Einheitszelle. Alle OH-Gruppen haben - wie bei $\mathrm{Li}(\mathrm{OH})$ - die gleiche Umgebung ${ }^{48}$, nach IR-Daten liegen nahezu lineare Wasserstoffbrücken der Länge $3,35 \AA$ vor ${ }^{49}$. Im Schwingungsspektrum sind Banden von maximal $2 \mathrm{OH}$-Valenzschwingungen und $4 \mathrm{OH}$-Librationsschwingungen bzw. OH-Deformationsschwingungen zu erwarten. Diese Schwingungen müssen - wegen der niedrigen Symmetrie des Kristallgitters - alle im IR-Spektrum erlaubt sein. Im IRSpektrum ${ }^{49,204,215}$ (Abb. 10) treten bei $23{ }^{\circ} \mathrm{C}$ eine etwas verbreiterte $\mathrm{OH}-$ Valenzschwingungsbande bei $3600 \mathrm{~cm}^{-1}$ und bei $-180{ }^{\circ} \mathrm{C} 2 \mathrm{OH}$-Valenzschwingungsbanden $\left(v_{\mathrm{OH}}\right)$ bei 3556 und $3533 \mathrm{~cm}^{-1}$ auf. Die Aufspaltung dieser Banden ist dabei auf DipolDipol-Kopplung der beiden OH-Gruppen in der Einheitszelle zurückzuführen. So zeigen verdünnte feste 


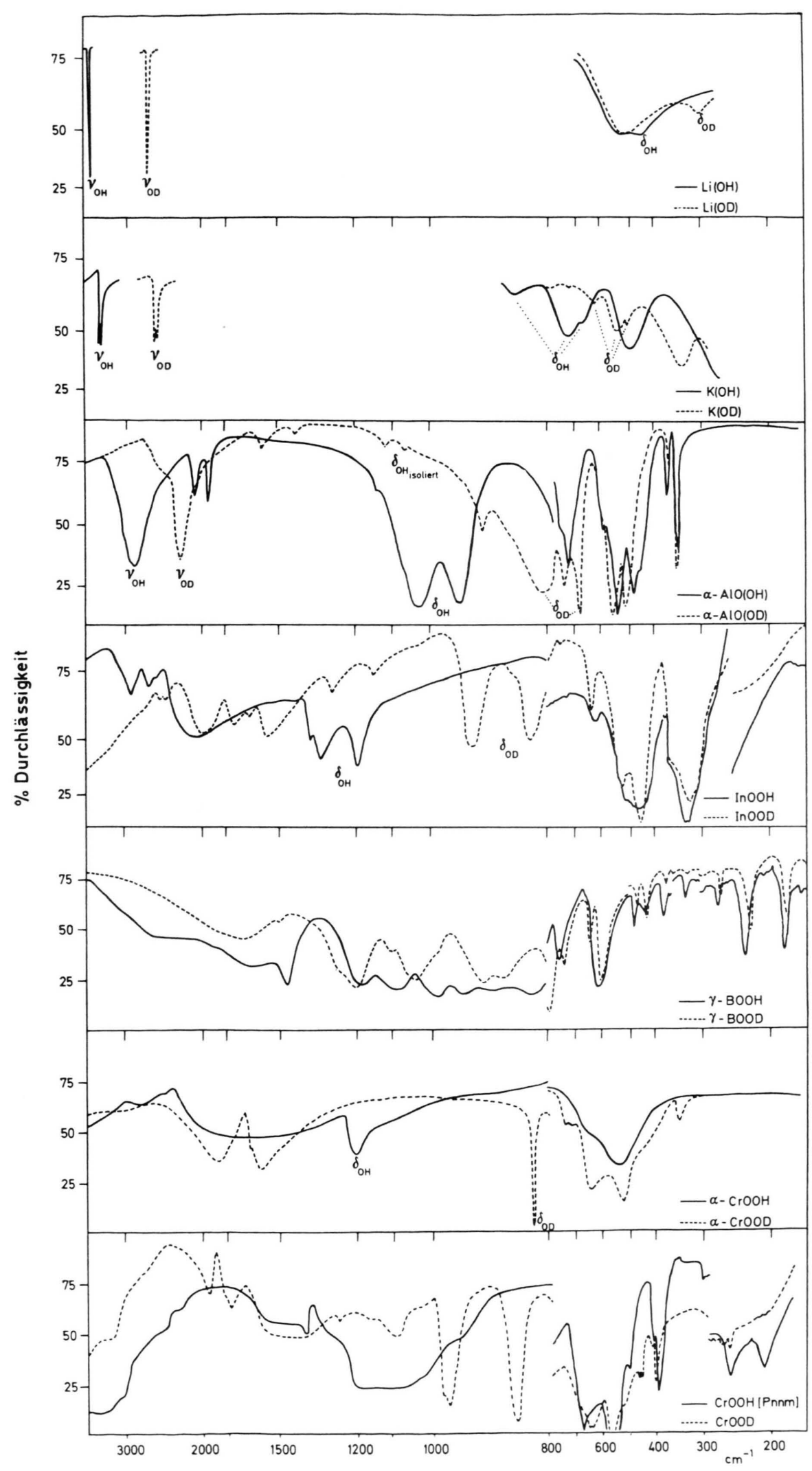

Abb. 10. IR-Absorptionsspektren (KBr- bzw. CsJ-Preßlinge) von polykristallinen Hydroxiden mit strukturell äquivalenten $\mathrm{OH}$-Gruppen und mit von oben nach unten zunehmender Wasserstoffbrücken-Bindungsstärke. 
Lösungen von $\mathrm{K}(\mathrm{OH})$ in $\mathrm{K}(\mathrm{OD})$ bei $-180{ }^{\circ} \mathrm{C}$ anstelle dieses Dubletts nur eine einzige Bande bei $3527 \mathrm{~cm}^{-1}$. In diesen partiell deuterierten Präparaten von ungefähr der Zusammensetzung $\mathrm{K}(\mathrm{OH})_{0,1}(\mathrm{OD})_{0,9}$ ist eine $\mathrm{OH}-\mathrm{Gruppe}$ statistisch nur noch von OD-Gruppen umgeben. Eine Wechselwirkung der $\mathrm{OH}$-Gruppen untereinander ist hier nicht mehr möglich, die zwischen $\mathrm{OH}$ - und OD-Gruppen nur gering. Man erhält so die Schwingungen der isolierten $\mathrm{OH}-\mathrm{Gruppe}^{49}$. Im langwelligen Infrarotbereich erscheinen bei $\mathrm{K}(\mathrm{OH})$ bei $23{ }^{\circ} \mathrm{C}$ eine breite Absorption bei $643 \mathrm{~cm}^{-1}$, bei $-180{ }^{\circ} \mathrm{C}$ drei Banden bei 840, 709 und $671 \mathrm{~cm}^{-1}$. Diese verschwinden beim Deuterieren und erscheinen um den Faktor 1,35 verschoben im noch ferneren IR-Bereich. Sie werden $\mathrm{OH}$-Deformationsschwingungen $\left(\delta_{\mathrm{OH}}\right)$ zugeordnet. In weitgehend deuterierten Präparaten treten neben den $\delta_{\mathrm{OD}}$-Banden anstelle der $3 \delta_{\mathrm{OH}^{-}}$ Banden 2 schwache Banden bei 765 und $670 \mathrm{~cm}^{-1}$ auf, die auf die 2 Deformationsschwingungen einer isolierten $\mathrm{OH}$-Gruppe zurückgeführt werden. Die Nähe der beiden Banden steht dabei im Einklang mit einem die freie Rotation der $\mathrm{OH}$-Gruppe verhinderndem Potential, das nahezu zylindrisch um die $\mathrm{O}-\mathrm{H}$-Bindung herumliegt und im wesentlichen von Wasserstoffbrückenbindung herrührt. Eine weitere Bande im IR-Spektrum von $\mathrm{K}(\mathrm{OH})$ bei 470 $\mathrm{cm}^{-1}$ verschiebt sich beim Deuterieren nur um den Faktor 1,22. Dies bedeutet, daß hier primär keine $\mathrm{OH}$-Deformationsschwingung vorliegt. Sie wird einer Translationsschwingung des Gitters zugeordnet, an der mehr Hydroxid- als Kalium-Bewegung beteiligt ist. Eine Absorption im noch ferneren Infrarot rührt von Transłationsschwingungen des Gitters her ${ }^{49}$. IR-Spektren wurden auch von den folgenden Hydroxiden mit schwacher Wasserstoffbrückenbindung untersucht: $\quad \beta$ - $\mathrm{Be}(\mathrm{OH})_{2}{ }^{230,}{ }^{231}$, $\mathrm{Al}(\mathrm{OH})_{3}{ }^{230},{ }_{232}, \quad \mathrm{Cu}(\mathrm{OH})_{2}{ }^{233}, \quad \varepsilon-\mathrm{Zn}(\mathrm{OH})_{2}{ }^{230,}{ }^{234}$, $\mathrm{Sr}(\mathrm{OH})_{2}{ }^{234-237}, \operatorname{In}(\mathrm{OH})_{3}{ }^{238}$ und $\mathrm{Ba}(\mathrm{OH})_{2}{ }^{237}$.

\section{a-AlO $(\mathrm{OH})$}

Ein Beispiel für Hydroxide mit mittelstarker Wasserstoffbrückenbindung ist Diaspor, $\alpha-\mathrm{AlO}(\mathrm{OH})$. Im IR-Spektrum dieser in der Raumgruppe Pbnm mit 4 Formeleinheiten kristallisierenden Verbindung sind Banden von maximal $2 \mathrm{OH}-$ Valenz- und $4 \mathrm{OH}$ Deformationsschwingungen $\mathrm{zu}$ erwarten. So wird cine Absorption bei etwa $2950 \mathrm{~cm}^{-1}$ im IR-Spektrum 233, 239-242 (Abb. 10) OH-Valenzschwingungen zugeordnet. Banden bei 1077 und $963 \mathrm{~cm}^{-1}$ wer- den auf $\mathrm{OH}$-Deformationsschwingungen, schwache Absorptionen bei 2120 und $1990 \mathrm{~cm}^{-1}$ auf die ersten Obertöne dazu, zurückgeführt. Diese Banden treten im IR-Spektrum von $\alpha$-AlO(OD) gesetzmäßig um etwa den Faktor $\sqrt{2}$ verschoben bei kleineren Wellenzahlen auf. Isostrukturell mit Diaspor sind $\alpha-\mathrm{ScO}(\mathrm{OH}), \alpha-\mathrm{FeO}(\mathrm{OH})$ und $\mathrm{GaO}(\mathrm{OH})$. Im Kristallgitter von $\alpha$-ScO(OH) liegt ein $\mathrm{O}(\mathrm{H}) \ldots \mathrm{O}$ Abstand von 2,73 $\AA$ vor ${ }^{80}$. Die Lage des Wasserstoffs ist unbekannt. Wegen des im Vergleich zum Diaspor größeren $\mathrm{O}(\mathrm{H}) \ldots \mathrm{O}$-Abstandes, was einer schwächeren Wasserstoffbrückenbindung entspricht, erfolgen im IR-Spektrum von $\alpha-\mathrm{ScO}(\mathrm{OH})$ die $\boldsymbol{\nu}_{\mathrm{OH}^{-}}$ Schwingung bei höheren Wellenzahlen $\left(3122 \mathrm{~cm}^{-1}\right)$ und die $\delta_{\mathrm{OH}^{-}}$Schwingungen bei kleineren Wellenzahlen (901 und $819 \mathrm{~cm}^{-1}$ ) ${ }^{80,}, 242$.

Bei $\alpha-\mathrm{FeO}(\mathrm{OH})$ erscheint die Kristallstruktur nicht genau genug bestimmt. Auf Grund seines IRSpektrums dürften in diesem Oxidhydroxid Bindungsverhältnisse in der Wasserstoffbrücke vorliegen, die denen in $\alpha-\mathrm{ScO}(\mathrm{OH})$ recht ähneln ${ }^{233,}$ 240, 242. Von $\mathrm{GaO}(\mathrm{OH})$ ist nur bekannt, daß es mit Diaspor isomorph ist. Sowohl die $\boldsymbol{v}_{\mathrm{OH}^{-}}$als auch die $\delta_{\mathrm{OH}}$-Banden zeigen in den Spektren beider Hydroxide in etwa gleicher Lage ${ }^{192,238,239,242,243}$. Trotz der strukturellen Äquivalenz aller Wasserstoffbrücken im Kristallgitter der Hydroxide mit Diasporstruktur ist in den IR-Spektren eine Aufspaltung der $\mathrm{OH}$ Deformations-Schwingungsbanden in jeweils 2 Banden erkennbar. Nach Messungen an partiell deuterierten Präparaten ist diese Aufspaltung z. T. auf eine Kopplung der Deformationsschwingungen der $\mathrm{OH}-G r u p p e n$ in der Einheitszelle zurückzuführen. So sind im fast vollständig deuterierten Präparat nur noch 2 schwache Banden in diesem Wellenzahlbereich vorhanden. Die Aufspaltung dieser beiden restlichen Banden beträgt nur noch etwa die Hälfte. Vermutlich sind diese beiden Banden auf die 2 Deformationsschwingungen einer isolierten OH-Gruppe im Kristallgitter des Deuteroxids zurückzuführen. Die beobachtete restliche Aufspaltung weist dabei auf eine leichte Unsymmetrie des Potentialfeldes, das um die OH-Bindung herumliegt, hin. Dieser Befund steht im Einklang mit Messungen im polarisierten IR-Strahl an orientierten Einkristallen von $\alpha-\mathrm{AlO}(\mathrm{OH})$ und $\alpha-\mathrm{FeO}(\mathrm{OH})$. Abb. 11 zeigt als Beispiel die Reflexion eines polarisierten IR-Strahls an der Fläche (110) eines Einkristalls von $\alpha-\mathrm{FeO}(\mathrm{OH})$.

Die Bande bei höheren Wellenzahlen rührt danach im wesentlichen von Schwingungen her, die un- 


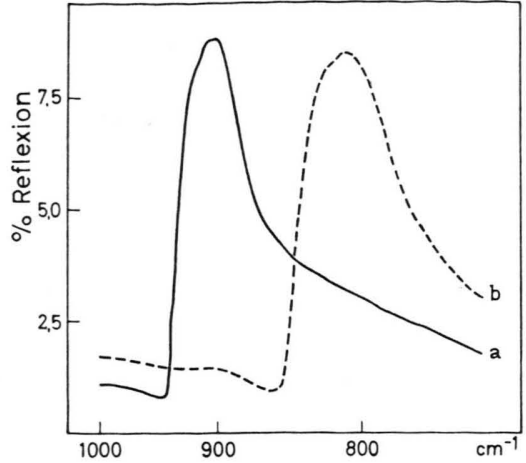

Abb. 11. IR-Reflexionsspektrum des $a \cdot \mathrm{FeO}(\mathrm{OH})$ von der Fläche (110). Kurve a: Polarisatorstellung \|[110]; Kurve b: Polarisatorstellung \|[001].

gefähr in der Spiegelebene verlaufen (vgl. Abb. 2) . Die Absorption bei niedrigen Wellenzahlen ist hauptsächlich auf OH-Deformationsschwingungen zurückzuführen, die in etwa senkrecht zur Spiegelebene erfolgen. Im fernen IR-Bereich im Spektrum von $\alpha-\mathrm{AlO}(\mathrm{OH})$ auftretende Banden verschieben sich beim Deuterieren praktisch nicht. Auch von den folgenden Hydroxiden mit mittelstarker Wasserstoffbrückenbindung sind die IR-Spektren untersucht worden: $\gamma-\mathrm{AlO}(\mathrm{OH})^{233}, 239,244-246$ und die damit isomorphen Verbindungen $\gamma-\mathrm{ScO}(\mathrm{OH})^{80}$, $\gamma-\mathrm{CrO}(\mathrm{OH})^{89}$ und $\gamma \cdot \mathrm{FeO}(\mathrm{OH})^{233,240,244} ; \beta-\mathrm{UO}_{2}$ $(\mathrm{OH})_{2}{ }^{112,247} ; \mathrm{Si}_{2} \mathrm{O}_{3}(\mathrm{OH})_{2}{ }^{93} ; \mathrm{U}_{2} \mathrm{O}_{5}(\mathrm{OH})_{2}{ }^{112}$.

\section{$\mathrm{InOOH}$}

In diesem Oxidhydroxid liegt eine starke Wasserstoffbrückenbindung vor. Es kristallisiert in der Raumgruppe Pnnm mit 2 Formeleinheiten in der Einheitszelle. Die im Kristallgitter vorliegenden O - O-Abstände von 2,58 $\AA$ werden als Wasserstoffbrücken gedeutet. Die Protonenlagen konnten bei der Röntgenstrukturanalyse nicht festgelegt werden ${ }^{100}$. Der Schluß auf das Vorliegen einer Raumgruppe mit Symmetriezentrum beruht dabei auf der Annahme, daß das Proton in der Wasserstoffbrücke entweder statisch oder dynamisch auf der Mitte dieser Brücke sitzt. Im IR-Spektrum von $\mathrm{InOOH}$ (Abb. 10) treten in jenem Bereich, in dem normalerweise Banden von $\mathrm{OH}-$ Valenzschwingungen zu erwarten sind, 3 Banden mit Maxima bei 2680, 2420 und $1920 \mathrm{~cm}^{-1}$ auf. InOOD zeigt 3 entsprechende verschobene Absorptionen mit anderer Intensitätsverteilung bei 1915, 1685 und $1475 \mathrm{~cm}^{-1}$. Das bei $-170^{\circ} \mathrm{C}$ aufgenommene IR-Spektrum von $\mathrm{InOOH}$ zeigt in diesem Bereich gegenüber dem bei
Raumtemperatur aufgenommenen Spektrum starke Intensitätsunterschiede. Die Tatsache, daß sich die Frequenzlage dieser Banden in partiell deuterierten Präparaten praktisch nicht verändert, schließt eine Erklärung für die Multiplizität dieser Banden durch Kopplung der Valenzschwingungen der beiden $\mathrm{OH}$ Gruppen in der Einheitszelle aus. Für die Bandenaufspaltung muß also eine andere Erklärung gesucht werden. Dazu käme einmal eine Wechselwirkung (F e rmi-Resonanz) von $\mathrm{OH}(\mathrm{OD})$-Valenzschwingungen mit ersten Obertönen bzw. Kombinationstönen von $\mathrm{OH}(\mathrm{OD})$-Deformationsschwingungen in Frage. Aber auch Tunnelprozesse in einem Doppelminimum-Potential dürften sicherlich einen weiteren Beitrag zur detaillierten Bandenform und Bandenlage dieser Absorptionen liefern. So wurden denn auch ${ }^{1} \mathrm{H}$-NMR-Breitlinienspektren im $\mathrm{Zu}$ sammenhang mit Doppelminimum-Potentialflächen für die $\mathrm{O}-\mathrm{H}-\mathrm{O}$-Bindung diskutiert. Bei $24{ }^{\circ} \mathrm{C}$ er. gibt sich eine einfache ${ }^{1} \mathrm{H}$-NMR-Absorptionskurve mit einem zweiten Moment von 7,37 Gauss ${ }^{2}$. Bei der Verfolgung der Abhängigkeit des zweiten Moments von der Temperatur zeigt es sich, daß das zweite Moment bei $-51{ }^{\circ} \mathrm{C}$ auf 8,10 Gauss $^{2}$ ansteigt. Von $-51{ }^{\circ} \mathrm{C}$ bis zu der tiefsten bei der Messung erreichten Temperatur von $-136{ }^{\circ} \mathrm{C}$ ist keine weitere Änderung des zweiten Moments zu beobach. ten. Die Zunahme des zweiten Moments wird auf eine diskontinuierliche Abnahme der Beweglichkeit der Protonen zurückgeführt. Nach D e b y e $\mathrm{Scher}$ rer-Aufnahmen ist bei $\mathrm{InOOH}$ zwischen $24{ }^{\circ} \mathrm{C}$ und $-140{ }^{\circ} \mathrm{C}$ eine Strukturumwandlung 1. Ordnung nicht erkennbar. Weitere Banden im IRSpektrum von $\mathrm{InOOH}$ zwischen 1300 und 1100 $\mathrm{cm}^{-1}$ werden im wesentlichen den OH-Deformationsschwingungen zugeordnet, die entsprechenden OD-Banden sind um einen Faktor von etwa $/ 2$ nach kleineren Wellenzahlen verschoben. Banden im noch ferneren IR-Bereich verschieben sich beim Deuterieren praktisch nicht, sie werden hauptsächlich auf Indium-O-Schwingungen zurückgeführt ${ }^{248,249}$. Ähnliche Verhältnisse liegen auch bei dem mit $\mathrm{InOOH}$ strukturell verwandten $\gamma-\mathrm{MnO}(\mathrm{OH})$ vor $233,240,241,258,259$.

\section{$\gamma-\mathrm{BOOH}$}

Nach einer Röntgenstrukturanalyse ${ }^{91}$ bilden in der kubischen Modifikation von Metaborsäure, $\gamma$-BOOH, die Bor- und die O-Atome ein dreidimensionales Netzwerk von $\mathrm{BO}_{4}$-Tetraedern mit Wasser- 
stoffbrücken zwischen den Tetraedern. Die Wasserstoffatome bilden eine starke Bindung $\mathrm{O}_{\mathrm{II}}-\mathrm{H}=$ $1,06 \AA$ und eine schwächere $\mathrm{H} \cdots \mathrm{O}_{\mathrm{I}}=1,43 \AA$. Die Länge der nahezu linearen Gruppe $\mathrm{O}_{\mathrm{II}}(\mathrm{H}) \ldots \mathrm{O}_{\mathrm{I}}$ beträgt 2,487 $\AA$. Parallel zu der $\mathrm{O}_{\mathrm{II}}-\mathrm{H} \cdots \mathrm{O}_{\mathrm{I}}$-Achse ergab sich ein sehr hoher thermischer Faktor für Wasserstoff entsprechend einer Wurzel aus der mittleren quadratischen Abweichung von $0,37 \pm 0,08 \AA$. Dieses Ergebnis deutet auf merkliche Resonanz mit der Konfiguration $\mathrm{O}_{\mathrm{II}} \cdots \mathrm{H}-\mathrm{O}_{\mathrm{I}}$ hin. $\gamma$-BOOH kristallisiert in der Raumgruppe $\mathrm{P} \overline{4} 3 \mathrm{n}$ mit 24 Formeleinheiten in der Einheitszelle. Alle $\mathrm{O}-\mathrm{H} \cdots \mathrm{O}$-Gruppen sind strukturell äquivalent. Im IR-Spektrum von $\gamma$-BOOH ${ }^{250,251}$ (Abb. 10) tritt im „OH-Valenzschwingungsbereich" eine sehr breite Absorption mit Maxima bei 3060,1650 und $1465 \mathrm{~cm}^{1}$ auf. Im IR-Spektrum von $\gamma$-BOOD erscheint dort eine breite Absorption mit einem Maximum bei $1700 \mathrm{~cm}^{-1}$. Anscheinend fallen weitere verschobene Banden in den Bereich der Gitterschwingungen (Schulterbanden bei 1260 und $1105 \mathrm{~cm}^{-1}$ ). Offensichtlich liegt eine enge Verknüpfung dieser Banden mit einer Bewegung von Protonen bzw. Deuteronen vor. Ban. den bei 990 und $827 \mathrm{~cm}^{-1}$ im IR-Spektrum von $\gamma$-BOOH und verschoben bei 790 und $633 \mathrm{~cm}^{-1}$ im IR-Spektrum von $\gamma$-BOOD sind wahrscheinlich hauptsächlich $\mathrm{OH}(\mathrm{bzw}$. $\mathrm{OH})$-Deformationsschwingungen zuzuordnen. Die dabei beobachtete normale $\mathrm{H}$ - D-Isotopenverschiebung liegt aber bei den Banden im „OH(bzw. OD)-Valenzschwingungsbereich“ nicht vor. Zudem sind dort die Intensitätsverhältnisse bei den OH- im Vergleich zu den OD-Bandengruppen grundlegend verschieden. Die Aufspaltung und Breite dieser Banden könnte auf Kopplung zwischen den $\mathrm{OH}-\mathrm{Gruppen}$ der Einheitszelle zurückzuführen sein. Versuche, die Valenzschwingungen einer von anderen Hydroxylgruppen isolierten $\mathrm{OH}$ Gruppe durch Aufnahme der IR-Spektren weitgehend deuterierter Präparate zu ermitteln, schlugen fehl. So bildete sich aus weitgehend deuterierter Orthoborsäure unter den Bedingungen der $\gamma$-BOOHSynthese auf Grund eines anomalen H-D-Isotopeneffektes nur die partiell deuterierte $\beta$-Modifikation von BOOH. Zur Deutung der Absorptionen im „OH(OD)-Valenzschwingungsbereich" käme ferner eine Wechselwirkung von $\mathrm{OH}(\mathrm{bzw}$. OD)-Valenzund $\mathrm{OH}$ (bzw. OD)-Deformationsschwingungen in Frage. Aber auch Effekte, die mit DoppelminimumPotentialflächen für die Bewegung der Wasserstoffisotopen in der Wasserstoffbrücke zusammenhängen, könnten hier eine Rolle spielen. So verhindert der anomale $\mathrm{H}-\mathrm{D}$-Isotopeneffekt die Bildung von $\gamma$-BOOD nach der Darstellungsmethode von $\gamma$-BOOH. Dieses abweichende Verhalten wird auf der Grundlage einer verschiedenen Dichte von $H$ und $D$ im Zentrum eines Doppelminimum-Potentials für die Wasserstoffbrückenbindung gedeutet ${ }^{252,253}$. Weitere Banden im IR-Spektrum von $\gamma$-BOOH und $\gamma$-BOOD werden hauptsächlich $\mathrm{B}-\mathrm{O}$-Schwingungen zugeordnet, Absorptionen im IR-Bereich $<400 \mathrm{~cm}^{-1}$ rühren vermutlich von den Valenzschwingungen der Wasserstoffbrücke $\underset{v_{0}-\mathrm{H} \cdots 0}{\rightarrow}$ bzw. $v_{0}-\mathrm{D} \cdots \stackrel{0}{\leftarrow}$, her ${ }^{251}$.

\section{$\mathrm{CrOOH}$ (Raumgruppe $\mathrm{R} \overline{3} \mathrm{~m}$ )}

Das rhomboedrische Chromoxidhydroxid $\alpha-\mathrm{CrOOH}$ kristallisiert in der Raumgruppe $R \overline{3} \mathrm{~m}$ mit einer Formeleinheit in der Einheitszelle ${ }^{103}$. Neutronenbeugungsaufnahmen ${ }^{104,105}$ und ${ }^{1} \mathrm{H}$-NMR-Breitlinienspektren ${ }^{106}$ ergaben, daß sich das Proton auf der Mitte der Wasserstoffbrücke der Länge 2,49 $\AA$ aufhält. Jedoch kann mit solchen Messungen eine Protonenbewegung in einem symmetrischen Doppelminimum-Potential nicht von einer Bewegung in einem symmetrischen Potential mit nur einem Minimum in der Mitte der Brücke unterschieden werden. Im IR-Spektrum 241, 254, 255 (Abb. 10) tritt eine sehr breite Bande mit einem Maximum bei $1650 \mathrm{~cm}^{-1}$ auf, die einer OH-Valenzschwingung zugeordnet wird. Im IR-Spektrum von $\alpha$-CrOOD treten dagegen zwei OD-Valenzschwingungsbanden bei 1600 und $1920 \mathrm{~cm}^{-1}$ auf. Die Potentialkurven für die $\mathrm{O}-\mathrm{H}-\mathrm{O}$ - und die $\mathrm{O}-\mathrm{D}-\mathrm{O}$-Bindungen sind also offensichtlich verschieden. Berechnungen darüber sind durchgeführt worden ${ }^{254}$. Danach befindet sich bei $\alpha-\mathrm{CrOOH}$ die Barriere zwischen den beiden Potentialminima in der $\mathrm{O}-\mathrm{H}-\mathrm{O}$-Bindung, sofern sie überhaupt vorhanden ist, unterhalb des Schwingungsniveaus im Grundzustand. Dagegen ist die Potentialbarriere in der $\mathrm{O}-\mathrm{D}-\mathrm{O}$-Bindung beträchtlich höher, vermutlich in der Nähe des dritten oder vierten angeregten Zustands. Somit ist vom Standpunkt des IR-Spektrums die $\mathrm{O}-\mathrm{H}-\mathrm{O}$-Bindung in $\alpha$-CrOOH symmetrisch, wohingegen die $\mathrm{O}-\mathrm{D}-\mathrm{O}$-Bindung in $\alpha$-CrOOD asymmetrisch ist. Dies erklärt auch den im Vergleich zum $\mathrm{O}(\mathrm{H}) \mathrm{O}$ Abstand um 0,06 längeren $\mathrm{O}$ (D) O-Abstand. Bei $1229 \mathrm{~cm}^{-1}$ tritt eine OH-Deformationsschwingungsbande auf, bei $832 \mathrm{~cm}^{-1}$ eine OD-Deformationsschwingungsbande, neben Banden von Chrom-Sauerstoff-Schwingungen im ferneren IR-Bereich. Sehr 
ähnliche Verhältnisse liegen bei dem mit $\alpha-\mathrm{CrOOH}$ isostrukturellen $\mathrm{CoOOH}$ vor ${ }^{260,}{ }^{261}$.

\section{$\mathrm{CrOOH}$ (Raumgruppe Pnnm)}

Von der in der Raumgruppe Pnnm mit 2 Formeleinheiten in der Einheitszelle kristallisierenden Modifikation des $\mathrm{CrOOH}$ sind die Lagen der Crund O-Atome mittels Röntgenbeugung an Pulver bestimmt worden ${ }^{102}$. Isomorph mit dieser Modifikation von $\mathrm{CrOOH}$ ist $\mathrm{InOOH}$. Alle $\mathrm{O}-\mathrm{H}-\mathrm{O}$ Gruppen sind strukturell äquivalent. Es tritt ein extrem kurzer $\mathrm{O}(\mathrm{H}) \mathrm{O}$-Abstand von $2,4 \pm 0,2 \AA$ auf. Die Lage der Protonen ist unbekannt. Die Struktur der wahrscheinlich in der Raumgruppe Pnnm kristallisierenden CrOOD-Modifikation ist noch nicht bestimmt worden. Röntgenaufnahmen nach dem Debye-Scherrer-Verfahren ergaben für dieses CrOOD ein dem in der Raumgruppe Pnnm auftretenden $\mathrm{CrOOH}$ sehr ähnliches Beugungsdiagramm ${ }^{256}$. Anscheinend sind die Synthesebedingungen von $\mathrm{CrOOH}$ und $\mathrm{CrOOD}$ etwas verschieden ${ }^{257}$. Im IR-Spektrum von $\mathrm{CrOOH}^{256,}{ }^{257}$ (Abb. 10) erscheint im „Bereich der $\mathrm{OH}-$ Valenz- und der $\mathrm{OH}$ Deformationsschwingungen " eine sehr breite Absorption. Wegen des Fehlens von diskreten Banden ist eine Zuordnung von Absorptionen zu OH-Valenzund $\mathrm{OH}$-Deformationsschwingungen offensichtlich nicht möglich. Das IR-Spektrum von CrOOD ist in diesem Bereich davon grundlegend verschieden, was mit einem normalen $\mathrm{H}$ - D-Isotopeneffekt nicht erklärt werden kann. Die bei $-170^{\circ} \mathrm{C}$ aufgenommenen IR-Spektren zeigen in diesem Bereich gegenüber den bei Raumtemperatur aufgenommenen Spektren starke Intensitätsunterschiede. Das IR-Spektrum von CrOOD ähnelt in bezug auf die Bandenlagen im Bereich der OD-Valenz- und der OD-Deformationsschwingungen dem IR-Spektrum von InOOD, im Hinblick auf die Bandenform dem IR-Spektrum von InOOH. Bei CrOOD dürften für die Wasserstoffbrücke ähnliche Bindungsverhältnisse wie bei $\mathrm{InOOH}$ vorliegen. Im fernen IR-Bereich auftretende Banden werden hauptsächlich Chrom-SauerstoffSchwingungen zugeordnet.

\section{Zusammenfassung}

Betrachtet man zusammenfassend diese typischen Beispiele von strukturell einfach aufgebauten $\mathrm{Hy}$ droxiden, bei denen die OH-Gruppen im Kristallgitter alle die gleiche Umgebung haben, so kann man folgendes feststellen: Bei Hydroxiden ohne Wasserstoffbrücken liegt die Wellenzahl der $\mathrm{OH}^{\circ}$. Valenzschwingung ungefähr im Bereich von 3700 bis $3600 \mathrm{~cm}^{-1}$, die Wellenzahl der $\mathrm{OH}^{\ominus}$-Librationsschwingung zwischen 300 und $500 \mathrm{~cm}^{-1}$. Das Auftreten von Wasserstoffbrückenbindung übt einen merklichen Einfluß auf die Schwingungen der $\mathrm{OH}$ Gruppen aus. Mit zunehmender Stärke dieser Bindung - mit abnehmendem $\mathrm{O}(\mathrm{H}) \ldots$. . Abstand wird die Wellenzahl der OH-Valenzschwingung erniedrigt, die entsprechenden Banden im IR-Spektrum werden immer breiter. Das Maximum der Absorption verschiebt sich von etwa 3600 bis $1600 \mathrm{~cm}^{-1}$. Gleichzeitig ändert sich für das Proton in der Wasserstoffbrücke immer mehr auch die Form der Fläche der potentiellen Energie, die - neben den Symmetrieverhältnissen in der primitiven Einheitszelle - die Zahl der IR-aktiven Schwingungen bedingt und einen zunehmenden Beitrag zur detaillierten Bandenform liefert. Dipol-Dipol-Kopplung der OH-Gruppen in der Einheitszelle und F e r m i - Resonanz zwischen Obertönen und Kombinationen von Gitterschwingungen, insbesondere dem ersten Oberton der OH(OD)-Deformationsschwingungen, und der $\mathrm{OH}(\mathrm{OD})$-Valenzschwingung dürfte ebenfalls zu komplizierten breiten $\mathrm{OH}(\mathrm{OD})$ Valenzschwingungsbanden führen. Messungen der IR-Spektren von partiell deuterierten Hydroxiden liefern Schwingungsbanden von isolierten OH-Gruppen, deren Bewegungen nicht mehr mit Bewegungen anderer strukturell äquivalenter $\mathrm{OH}(\mathrm{OD})$-Gruppen in der Einheitszelle koppeln. Die Wellenzahl der $\mathrm{OH}$-Deformationsschwingung wird mit abnehmendem $\mathrm{O}(\mathrm{H}) \ldots \mathrm{O}$-Abstand erhöht. Bei sehr starker Wasserstoffbrückenbindung sind die Potentialflächen für die $\mathrm{O}-\mathrm{H}-\mathrm{O}$ - und die $\mathrm{O}-\mathrm{D}-\mathrm{O}$-Bindung verschieden. Aus der Lage der $\mathrm{OH}-\mathrm{V}$ alenzschwingungsbande im IR-Spektrum läßt sich - allerdings nur größenordnungsmäßig - die Länge des $0 \ldots O$. Abstandes in der Wasserstoffbrücke entnehmen. Aus Anzahl, Wellenzahl und Intensität der $\mathrm{OH}-$ Valenzschwingungsbanden läßt sich in sehr günstig gelagerten Fällen der Typ der Potentialfläche für die Bewegung des Protons bzw. Deuterons in der Wasserstoffbrücke ableiten, woraus die Symmetrie dieser Brücke folgt. Diese hier bei Hydroxiden mit nur strukturell äquivalenten OH-Gruppen angestellten Betrachtungen können nun auch auf die anderen Hydroxide mit verschiedenen OH-Gruppen in der Einheitszelle und auf Verbindungen, die Wasser- 
moleküle oder hydratisierte Protonen im Kristallgitter enthalten, angewandt werden.

Herrn Professor Dr. O. Glemser danke ich sehr für die Förderung dieser Arbeit, der Deutschen Forschungsgemeinschaft und dem Fonds der Chemischen Industrie für ihre Unterstützung.

1 O. Glemser, Angew. Chem. 73, 785 [1961].

2 H. E. Petch, Canad. J. Physics 35, 983 [1957].

3 H. E. Petch, Physic. Rev. 99, 1635 [1955].

4 J. D. Bernal u. H. D. Megaw, Proc. Roy. Soc. [London], Ser. A 151, 384 [1935].

5 H. E. Petch u. H. D. Megaw, J. opt. Soc. America 44, 744 [1954].

6 R. S. Mitchell, Z. Kristallogr. 125, 272 [1967].

7 W. R. Busing u. H. A. Levy, J. chem. Physics 26, 563 [1957].

8 H. E. Petch, Acta cristallogr. [Copenhagen] 14, 950 [1961].

9 D. M. Henderson u. H. S. Gutowsky, Amer. Mineralogist 47, 1231 [1962]

10 W. I. KWhiwidse u. L. N. Raschкowitsch, Ber. Akad. Wiss. UdSSR 146, 1322 [1962].

11 G. Aminoff, Z. Kristallogr. 56, 506 [1921].

12 H. Boutin u. W. Bassett, Amer. Mineralogist 48, 659 [1963].

13 F. Zigan u. R. Rothbauer, Neues Jb. Mineralog., Mh. 1967, 137.

14 D. D. Elleman u. D. Williams, J. chem. Physics 25, 742 [1956].

15 A. N. Christensen, Acta chem. scand. 19, 1765 [1965].

16 G. Natta u. E. Casazza, Rend. Acc. Naz. Lincei 5, 803 [1927].

17 W. Lotmar u. W. Feitknecht, Z. Kristallogr. 93, 386 [1936].

18 W. Feitknecht u. W. Bedert, Helv. chim. Acta 24, 676 [1941].

19 R. W. Cairns u. E. Ott, J. Amer. chem. Soc. 55, 527 [1933].

20 W. Feitknecht, Helv. chim. Acta 21, 766 [1938].

21 W. Feit K NECHT, Kolloid-Z. 92, 257 [1940].

22 W. FeitKneCht, Kolloid-Z. 93, 67 [1940].

23 T. ERnst, Z. physik. Chem. B 20, 65 [1933].

24 H. DACHS, Z. Kristallogr. 112, 60 [1959].

25 T. ChibA, J. chem. Physics 47, 1592 [1967].

26 T. Chiba, J. chem. Physics 47,5461 [1967].

27 T. ERnst, Nachr. Akad. Wiss. Göttingen, math.-phys. Kl., math.-physik.-chem., Abt. 1946, 76.

28 H. STEHR, Z. Kristallogr. 125, 332 [1967].

29 W. R. Busing, J. chem. Physics 23, 933 [1955].

30 O. Glemser, U. Hauschild u. H. Richert, Z. anorg. Chem. 290, 58 [1957].

31 R. S. Mitchell, Z. Kristallogr. 123, 459 [1966].

32 K. Schubert u. A. Seitz, Z. Naturforschg. 1, 321 [1946].

33 R. Fricke u. A. Seitz, Z. anorg. Chem. 254, 107 [1947].

34 P. W. Klewzow, R. F. Klewzowa u. L. P. Scheina, J. Struct. Chem. [UdSSR] 5, 583 [1964].

35 R. Fricke u. W. Dürrwächter, Z. anorg. Chem. 259, 305 [1949].

36 A. N. Christensen, R. G. Hazell u. A. Nilsson, Acta chem. scand. 21, 481 [1967].

37 R. Roy u. H. A. McKinstry, Acta cristallogr. [Copenhagen] 6, 365 [1953].

38 W. H. Zachariasen, Acta cristallogr. [Copenhagen] 1, 265 [1948].

39 W. H. Zachariasen, J. chem. Physics 16, 254 [1948].
40 P. W. Klewzow u. L. P. Sheina, Izvest. Akad. Nauk SSSR, Ser. neorgan. Mater. 1, 912 [1965].

41 M. Atoji u. D. E. Williams, J. chem. Physics. 31, 329 [1959].

42 R. C. Rav u. W. J. Glover JR., J. Amer. ceram. Soc. 47, 382 [1964].

43 R. F. Klevtsova u. P. V. Klevtsov, J. Struct. Chem. 5, 795 [1964].

44 A. N. Christensen, Acta chem. scand. 19, 1504 [1965].

45 A. N. Christensen, Acta chem. scand. 20, 2658 [1966].

46 P. V. Klevtsov u. L. P. Sheina. Izv. Akad. Nauk. SSSR. Neorg. Materialy 1, 2219 [1965].

47 A. N. Christensen, Acta chem. scand. 19, 1391 [1965].

48 J. A. Ibers, J. Kumamoto u. R. G. Snyder, J. chem. Physics 33, 1164 [1960].

49 R. G. Snyder, J. Кumamoto u. J. A. Ibers, J. chem. Physics 33, 1171 [1960].

50 W. C. Hamilton u. J. A. Ibers, Hydrogen Bonding in Solids, W. A. Benjamin, Inc., New York, Amsterdam 1968.

51 A. Seitz, U. Rösler u. K. Schubert, Z. anorg. Chem. 261, 94 [1950].

52 H. G. Schnering, Z. anorg. Chem. 330, 170 [1964].

53 R. B. Corey u. R. W. G. Wyckoff, Z. Kristallogr. A 86, 8 [1933].

54 A. BezjaK u. I. Jelenić, Acta Cryst. 16 Suppl., A 45 [1963].

55 G. Yamaguchi u. K. Sakamoto, Bull. chem. Soc. Japan 31, 140 [1958].

56 G. Yamaguchi u. K. SaKamoto, Can. J. Chem. 38, 1395 [1960].

57 K. SASVÁri, Magyar Kémiai Folyóirat 61, 314 [1955].

58 R. Rothbauer, F. Zigan u. H. O'Daniel, Z. Kristallogr. 125, 317 [1967].

59 H. SAalfeld, Neues Jb. Mineralog. 95, 1 [1960].

60 H. D. Megaw, Z. Kristallogr. 87, 185 [1934].

61 H. SaAlfeld u. B. B. Mehrotra, Naturwissenschaften 53, 128 [1966].

62 H. STRUNZ, Naturwissenschaften 52, 493 [1965].

63 A. N. Christensen u. N. C. Broch, Acta chem. scand. 21, 1046 [1967].

64 K. Schubert u. A. Seitz, Z. anorg. Chem. 256, 226 [1948].

65 A. Palm, J. physic. Colloid Chem. 52, 959 [1948].

66 A. D. Genkin u. I. V. Muraveva, Zapiski Vses. Min. Obshch. 92, 445 [1963].

67 H. D. Lutz, Z. Naturforschg. 20 b, 491 [1965].

68 H. Bärnighausen u. J. Weidlein, Acta cristallogr. [Copenhagen] 19, 1048 [1965].

69 M. Michaud, Rev. Chim. minérale 5, 89 [1968].

70 P. Buck u. H. Bärnighausen, Acta cristallogr. [Copenhagen] $\mathrm{B} 24,1705$ [1968].

71 H. R. Oswald u, H. JaGGi, Chimia 14, 22 [1960].

72 H. JAGGi u. H. R. Oswald, Acta cristallogr. [Copenhagen] 14, 1041 [1961].

73 W. FEITKNECHT, Chimia 11, 166 [1957].

74 P. M. DE Wolff, Acta cristallogr. [Copenhagen] 21, 432 [1966].

75 P. M. DE WolfF, Acta cristallogr. [Copenhagen] 22, 441 [1967].

76 W. R. Busing u. H. A. Levy, Acta cristallogr. [Copenhagen] 11, 798 [1958].

77 W. Hoppe, Z. Kristallogr. A 103, 73 [1941].

78 W. Hoppe, Z. Kristallogr. 104, 11 [1942].

79 J. Böhm u. G. Kahan, Z. anorg. Chem. 238, 350 [1938].

80 A. N. Christensen u. S. J. Jensen, Acta chem. scand. 21, 121 [1967].

81 H. T. Evans, JR. u. S. Block, Amer. Mineralogist 38, 1242 [1953]. 
${ }^{82}$ H. T. Evans, Jr. u. M. E. Mrose, Amer. Mineralogist 40, 861 [1955].

83 R. L. Collin u. W. N. Lipscomb, Acta cristallogr. [Copenhagen] 2, 104 [1949].

84 L. S. Dent Glasser u. L. Ingram, Acta cristallogr. [Copenhagen] B 24, 1233 [1968].

85 W. O. Milligan u. J. L. McAtee, J. physic. Chem. 60, 273 [1956].

86 P. P. Reichertz u. W. J. Yost, J. chem. Physics 14, 495 [1946].

76 C. H. Holm, C. R. Adams u. J. A. Ibers, J. physics Chem. 62, 992 [1958].

88 H. Auer-Welsbach u. K. Seifert, Mh. Chem. 93, 1110 [1962].

89 F. Hund, Naturwissenschaften 46, 320 [1959].

90 F. J. Ewing, J. chem. Physics 3, 420 [1935].

91 W. H. ZaChariasen, Acta cristallogr. [Copenhagen] 16 , 380 [1963].

92 F. Liebau u. F. Wodtke, Forschr. Mineralog. 39, 333 [1961].

93 F. Liebau, Z. Kristallogr. 120, 427 [1964].

94 E. Thilo, K.-H. Jost u. H. Worzala, Angew. Chem. 76, 714 [1964].

95 K.-H. Jost, H. Worzala u. E. Thilo, Acta cristallogr. [Copenhagen] 21, 808 [1966].

96 H. T. Evans, JR. u. M. E. Mrose, Amer. Mineralogist 45, 1144 [1960].

97 H. T. Evans, JR. u. M. E. Mrose, Acta cristallogr. [Co penhagen] 11, 56 [1958].

98 H. T. Evans, Z. Kristallogr. 116, 482 [1961].

99 M. E. Thompson, C. H. Roach u. R. Meyrowitz, Amer. Mineralogist 42, 455 [1957].

100 A. N. Christensen, R. Grønboeck u. S. R. Rasmussen, Acta. chem. scand. 18, 1261 [1964].

101 N. C. Tombs, W. J. Croft, J. R. Carter u. J. F. FitzGERALD, Inorg. Chem. 3, 1791 [1964].

102 A. N. Christensen, Inorg. Chem. 5, 1452 [1966]

103 R. M. Duglass, Acta cristallogr. [Copenhagen] 10, 423 [1957].

104 W. C.Hamilton u. J. A. Ibers, Acta cristallogr. [Copenhagen] 16, 1209 [1963].

105 W. C. Hamilton u. J. A. Ibers, Proc. physic. Soc. Japan 17, Suppl. B-II, 383 [1962].

106 J. A. Ibers, C. H. Holm u. C. R. Adams, Physic. Rev. 121, 1620 [1961].

107 I. D. Kondrachev u. N. N. Fedorova, Doklady Akad. Nauk SSSR 94, 229 [1954].

108 M. J. Buerger, Z. Kristallogr. 95, 163 [1936].

109 H. DACHS, Z. Kristallogr. 118, 303 [1963].

110 H. DACHS ,J. physic. Soc. Japan 17, 387 [1962].

111 J. K. Dawson, E. Wait, K. Alcock u. D. R. Chilton, J. chem. Soc. [London] 1956, 3531.

112 E. Schwarzmann u. O. Glemser, Z. anorg. Chem. 315 , 305 [1962].

113 G. Bergström u. G. Lundgren, Acta chem. scand. 10, 673 [1956].

114 R. B. Roof, Jr., D. T. Cromer u. A. C. Larson, Acta cristallogr. [Copenhagen] 17, 701 [1964].

115 A. L. Porte, H. S. Gutowsky u. J. E. Boggs, J. chem. Physics 36, 1700 [1962].

116 K. W. Bagnall u. J. B. Laidler, J, chem. Soc. [London] 1964, 2693.

117 W. H. Zachariasen, Acta cristallogr. [Copenhagen] 7, 305 [1954].

118 B. M. Craven u. T. M. Sabine, Acta cristallogr. [Copenhagen] 20, 214 [1966].

119 J. M. Cowley, Acta cristallogr. [Copenhagen] 6, 522 [1953].

120 J. M. Cowley, Nature [London] 171, 440 [1953].
121 K. Kume u. Y. Kakiuchi, J. physic. Soc. Japan 15, 1277 [1960].

122 J. A. Ibers u. C. H. Holm, J. physic. Soc. Japan 16, 839 [1961].

123 K. Kume u. Y. KakiUchi, J. physic. Soc. Japan 16, 843 [1961].

124 D. F. Hornig u. R. C. Plumb, J. chem. Physics 26, 637 [1957].

125 S. Furberg, Acta chem. scand. 9, 1557 [1955].

126 S. Burberg, Acta chem. scand. 8, 532 [1954].

127 F. E. Cole, Diss. Abs. 27 B, 1850 [1966].

128 R. PASCARD, Acta cristallogr. [Copenhagen] 7, 638 [1954].

129 R. PasCard, C. R. hebd. Séances Acad. Sci. 240, 2162 [1955].

130 C. Pascard-Billy, Acta cristallogr. [Copenhagen] 18, 827 [1965]

131 A. F. WELLS, Structural Inorganic Chemistry, 3. Aufl., Oxford at the Clarendon Press 1962.

132 M. T. Rogers u. L. Helmholz, J. Amer. chem. Soc. 63, 278 [1941].

133 A. Halász, A. Jánosi u. K. Lábdy, Veszpremi Vegyi pari Egyetem Köz. 4, 159 [1961].

134 B. S. Garrett, Oak Ridge Nat. Lab. Report Nr. 1745.

135 C. R. Peters u. M. E. Milberg, Acta cristallogr. [Copenhagen] 17, 229 [1964].

136 C. A. Coulson, Acta cristallogr. [Copenhagen] 17, 1086 [1964].

137 V. Luzzati, Acta cristallogr. [Copenhagen] 4, 120 [1951].

138 M. Bailey u. A. F. Wells, J. chem. Soc. [London] 1951, 968.

139 A. F. Wells u. M. Bailey, J. chem. Soc. [London] 1949, 1282.

140 L. M. Kirk patrick u. L. Pauling, Z. Kristallogr. 63, 502 [1926].

140a G. BAyer, J. less.-common Metals [Amsterdam] 16, 215 [1968].

141 Y. D. Feikema, Acta cristallogr. [Copenhagen] 14, 315 [1961].

142 O. JaRCHOw, Chem. Zvesti 13, 741 [1959].

143 Y. D. Feikema, Acta cristallogr. [Copenhagen] 20, 765 [1966].

144 Y. D. Feikema u. A. Vos, Acta cristallogr. [Copenhagen] 20, 769 [1966].

145 D. E. C. Corbridge u. E. J. Lowe, J. chem. Soc. [London] 1954, 493 und 4555 .

146 S. Furberg u. P. Landwark, Acta chem. scand. 11, 1505 [1957].

147 H. Beyer, O. Glemser u. B. Krebs, Angew. Chem. 80, 286 [1968].

148 S. Maričić u. J. A. S. Smith, J. chem. Soc. [London] 1958, 886.

149 S. Maričić u. J. A. S. Smith, Acta chem. scand. 10, 1362 [1956].

150 E. Schwarzmann u. O. Glemser, Z. anorg. Chem. 312 , 45 [1961].

151 W. Ja. Kabanov u. W. F. Tschuwajew, J. physik. Chem. [UDSSR] 38, 1317 [1964].

152 I. Lindeuist, Acta chem. scand. 4, 650 [1950].

153 I. LindQuist, Acta chem. scand. 10, 1362 [1956].

154 F. S. Lee u. G. B. Carpenter, J. physic. Chem. 63, 279 [1959].

155 C. E. Nordman, Acta cristallogr. [Copenhagen] 15, 18 [1962].

156 M. R. Truter, Acta cristallogr. [Copenhagen] 14, 318 [1961].

157 H. G. SMITH u. H. A. LeVy, Abstract für ACA meeting [1959]. Zitiert in l. c. ${ }^{156}$. 
158 D. E. Bethell u. N. Sheppard, J. chem. Physics 21, 1421 [1953].

159 E. R. ANdrew u. N. D. Finch, Proc. physic. Soc. 70 B, 980 [1957].

160 C. M. Gable, H. F. Betz u. S. H. Maron, J. Amer. chem. Soc. 72, 1445 [1950].

161 H. SaAlfeld, H. Matthies u. S. K. Datta, Ber. dtsch. keram. Ges. 45, 212 [1968].

162 I. Olovsson, J. chem. Physics 49, 1063 [1968].

163 A. F. Beecham, A. C. Hurley, M. F. Mackay, V. W. Maslen u. Mc L. Mahtieson, J. chem. Physics 49, 3312 [1968].

164 H. Rabaud u. R. Gay, Bull. Soc. franç. Minéralog. Cristallogr. 80, 166 [1957].

165 H. Rabaud, C. R. hebd. Séances Acad. Sci. 241, 1959 [1955].

166 R. Pepinsky, Z. Kristallogr. A 102, 119 [1940].

167 J. A. Wunderlich, Bull. Soc. franç. Minéralog. Cristallogr. 81, 287 [1958].

168 J. Clastre, Acta cristallogr. [Copenhagen] 7, 638 [1954].

169 H. Bärnighausen, Z. anorg. Chem. 342, 233 [1966].

170 H. G. Sмiтн, Acta cristallogr. [Copenhagen] 6, 604 [1953].

171 A. Preisinger, Tschermaks mineralog. petrogr. Mitt. 5, 123 [1954].

172 H. Manohar u. S. Ramaseshan, Current Sci. 32, 248 [1963].

173 W. H. Zachariasen, Acta cristallogr. [Copenhagen] 16, 385 [1963].

173a D. Mootz, J. Goldmann u. H. Wunderlich, Angew. Chem. 81, 116 [1969].

174 H. Worzala, Acta cristallogr. [Copenhagen] 24 B, 987 [1968].

175 R. Paetzold u. H. Amoulong, Z. Elektrochem. Ber. Bunsenges. physik. Chem. 65, 708 [1961].

176 P. Bourre-Maladiere, C. R. hebd. Séances. Acad. Sci. 246, 1063 [1958].

177 A. L. Porte, H. S. Gutowsky u. J. E. Boggs, J. chem. Physics 36, 1695 [1962].

178 P. G. Dickens u. R. Hurditch, Nature [London] 215, 1266 [1967].

179 M. J. Sienko u. H. Oesterreicher, J. Amer. chem. Soc. 90, 6568 [1968].

180 O. Glemser u. C. Naumann, Z. anorg. Chem. 265, 288 [1951].

181 O. Glemser, J. Weidelt u. F. Freund, Z. anorg. Chem. 332, 299 [1964].

182 O. Glemser u. G. Lutz, Z. anorg. Chem. 264, 17 [1951].

183 O. Glemser, U. Hauschild u. G. Lutz, Z. anorg. Chem. 269, 93 [1952].

184 O. Glemser, Nachr. Akad. Wiss. Göttingen, math. phys. Kl., Abt. II a, 121 [1955].

185 O. Glemser, G. Lutz u. G. Meyer, Z. anorg. Chem. 285, 173 [1956].

186 L. Kihlborg, G. Hägerström u. A. Rönnquist, Acta chem. scand. 15, 1187 [1961].

187 E. SchwarzmanN, Naturwissenschaften 49, 103 [1962].

188 E. Schwarzmann u. O. Glemser, Z. anorg. Chem. 315, 305 [1962].

189 E. SCHWARZMANN, Habilitationsschrift, Göttingen 1965.

190 E. Schwarzmann u. H. Marsmann, Naturwissenschaften 53, 349 [1966].

191 E. Hartert u. O. Glemser, Naturwissenschaften 40, 199 [1953].

192 E. Hartert u. O. Glemser, Naturwissenschaften 40, 552 [1953].

193 O. Glemser u. E. Hartert, Z. anorg. Chem. 283, 111 [1956].
194 E. Hartert u. O. Glemser, Z. Elektrochem. Ber. Bunsenges. physik. Chem. 60, 746 [1956].

194a R. Savoie u. P. A. Giguere, J. chem. Physics 41, 2698 [1964].

195 G. Zundel u. H. Metzger, Z. physik. Chem. [Frankfurt] 58, 225 [1968].

196 R. D. Gillard u. G. Wilkinson, J. chem. Soc. [London] 1964, 1640.

197 M. J. Sienko u. H. Oesterreicher, J. Amer. chem. Soc. 90, 6568 [1968].

198 R. L. SomorJaI, Dissertation, Princeton University 1963.

199 E. A. Pshenichov u. N. D. Sokolov, Opt. i. Spectroskopiya 17, 344 [1964].

200 A. F. Beecham, A. C. Hurley, M. F. Mackay, V. W. Maslen u. A. McL. Mathieson, J. chem. Physics 49, 3312 [1968].

201 C. Reid, J. chem. Physics 30, 182 [1959].

202 K. A. Wickersheim, J. chem. Physics 31, 863 [1959].

203 R. A. Buchanan, H. H. Caspers u. H. R. Marlin, J. chem. Physics 40, 1125 [1964].

204 R. A. Buchanan, J. chem. Physics 31, 870 [1959].

205 S. S. Mitra, Solid State Physics, F. Seitz u. D. TurnBull (Herausgeber), Academic Press, New York 1962 , 13,1 .

206 C. K. Coogan, J. chem. Physics 43, 823 [1965].

207 R. M. Hexter, J. chem. Physics 34, 941 [1961].

208 L. H. Jones, J. chem. Physics 22, 217 [1954].

209 R. A. Buchanan, E. L. Kinsey u. H. H. Caspers, J. chem. Physics 36, 2665 [1962].

210 R. M. Hexter, J. chem. Physics 38, 1024 [1963].

211 R. A. Buchanan u. H. H. Caspers, J. chem. Physics 38, 1025 [1963].

212 A. I. Stehcanow u. J. A. Popowa, Optics and Spectroscopy 11, 203 [1961].

213 J. Greenberg u. L. J. Hallgren, J. chem. Physics 35, 180 [1961].

214 B. A. Phillips u. W. R. Busing, J. physic. Chem. 61, 502 [1957].

215 D. Krishnamurti, Proc. Indian Acad. Sci. A 1959, 50.

216 Y. TA, C. R. hebd. Séances Acad. Sci. 221, 467 [1940].

217 C. Duval u. J. Lecomte, Bull. Soc. chim. France 8, 713 [1941] ; Bull. Soc. franç. Minéralog. Cristallogr. 66,284 [1943].

218 R. T. Mara u. G. B. B. M. Sutherland, J. opt. Soc. Amer. 43, 1100 [1953].

219 S. Mitra, Z. Kristallogr. 116, 149 [1961].

220 H. A. Benesi, J. chem. Physics 30, 852 [1959].

221 R. M. Hexter, J. opt. Soc. Amer. 48, 770 [1958].

222 R. A. Buchanan, H. H. Caspers u. J. Murphy, Appl. Optics 2, 1147 [1963].

223 R. T. Mara u. G. B. B. M. Sutherland, J. opt. Soc. Amer. 46, 464 [1956].

224 S. S. Mitra, Crystallography and Crystal Perfection,. Proc. Sympos. Madras, Academic Press Inc. Ltd., London 1963 , p. 347.

225 G. Safford, V. Brajovic u. H. Boutin, J. physic. Chem. Solids 24, 771 [1963].

226 I. Pelah, K. Krebs u. Y. Imry, J. chem. Physics 43, 1864 [1965].

227 W. R. Busing u. H. W. Morgan, J. chem. Physics 28, 998 [1958].

228 O. Oehler u. H. H. Günthard, J. chem. Physics 48, 2036 [1968].

229 P. V. Klevtsov, R. F. Klevtsova u. L. P. Sheina, J. Struct. Chem. 8, 229 [1967].

230 E. SchwarzmanN, Z. anorg. Chem. 317, 176 [1962].

231 I. J. Bear, G. M. Lukaszewsi u. A. G. Turnbull, Austral. J. Chem. 18, 1317 [1965]. 
232 V. A. Kolesova u. Y. I. RYskin, Optics and Spectroscopy 7,165 [1959].

233 C. Cabannes-Ott, Ann. Chimie XIII 5, 905 [1960].

234 O. K. Srivastava u. E. A. Secco, Can. J. Chem. 45, 585 [1967].

235 T. Dupuis, Mikrochim. Ichnoanalyt. Acta 1963, 976.

236 T. Dupuis, C. R. hebd. Séances Acad. Sci. 257, 405 [1963].

237 E. Schwarzmann u. L. Lange, Publikation in Vorbereitung.

238 T. Dupuis, Mikrochim. Ichnoanalyt. Acta 1964, 228.

239 V. A. Kolesova u. Y. I. Ryskin, J. Struct. Chem. 3, 656 [1962].

240 C. Cabannes-Ott, C. R. hebd. Séances Acad. Sci. 244, 2491 [1967].

241 E. Schwarzmann u. H. Marsmann, Naturwissenschaften 53, 349 [1966].

242 E. Schwarzmann u. H. Sparr, Z. Naturforschg. 24 b, 8 [1969].

243 A. Cornelis-Benoit, Spectrochim. Acta [London] 21, 623 [1965].

244 K. A. Wickersheim, J. chem. Physics 42, 579 [1965].

245 E. Jungmann, K. Klarić, S. Maričić u. Z. Meić, Symposium sur les Bauxites, Oxydes et Hydroxydes d'Aluminium, Zagreb 1963.
246 J. J. Fripiat, H. Bosmans u. P. G. Rouxhet, J. physic. Chem. 71, 1097 [1967].

247 A. M. DEANE, J. Inorg. nuclear Chem. 21, 238 [1961].

248 E. Schwarzmann, O. Glemser u. H. Marsmann, Naturwissenschaften 52, 344 [1965].

249 E. Schwarzmann, O. Glemser u. H. Marsmann, Z. Naturforschg. 21 b, 1128 [1966].

250 J. L. Parsons, A. H. Silver u. M. E. Mrlberg, J. chem. Physics 34, 2192 [1961].

251 E. Schwarzmann, Publikation in Vorbereitung.

252 E. Schwarzmann u. G. Christoph, Z. Naturforschg. 23 b, 1542 [1968].

253 E. Schwarzmann u. G. Christoph, Z. Naturforschg., im Druck.

254 R. G. SNyder u. J. A. Ibers, J. chem. Physics 36, 1356 [1962].

256 E. Schwarzmann u. L. Lange, Z. Naturforschg. 23 b. 874 [1968].

257 L. LANGE, Dissertation, Göttingen 1968.

258 E. Schwarzmann u. H. Marsmann, Z. Naturforschg. 20 b, 1124 [1965].

259 E. SChwarzmanN u. H. Marsmann, Z. Naturforschg. 21 b, 924 [1966].

260 A. BenOIT, Spectrochim. Acta [London] 19, 386 [1963].

261 A. BenOIT, Spectrochim. Acta [London] 19, 2011 [1963].

\title{
Studies on beta-Lactams
}

\author{
Basanta G. Chatterjee and Riaz F. Abdulla
}

Department of Applied Chemistry, Indian Institute of Technology, Kharagpur, W. Bengal, India

(Z. Naturforschg. 24 b, 1120-1127 [1969] ; eingegangen am 13. Mai 1969)

Since the discovery ${ }^{11}$ of the activating effect of the $\mathrm{N}$-( $p$-Nitro-phenyl)-group on the methine hydrogen of halogenacetamino esters, it has been found that the $p$-Acetyl-phenyl and $m$-Nitro-phenyl groups exert a similar effect. It has been shown that the delocalisation of the N-non-bonded electrons across the orbitals of the $N$-Aryl substituent is the governing factor in the cyclisation of the above systems, and the effect of the bulk of the $N$-Aryl substituent is relegated to a minor position.

\section{Discussion}

After the presence of the beta-lactam ring and its biological significance was established in Penicillin ${ }^{1}$, Cephalosporin and Pachysandra Terminalis ${ }^{2,3}$, several new methods were developed for its synthesis ${ }^{4}$. Among these, the intra-molecular alkylation of $N$-substituted halogen acetamino malonates seemed to be the most general ${ }^{5,6}$. The technique of SHEE-

1 H. T. Clarke, J. R. Johnson and R. Robinson, The Chemistry of Penicillin, Princeton University Press, Princeton 1947.

2 E. P. Abraham and G. G. F. Newton, Biochem. J. 79, 377 [1961].

3 T. KiKUCHI and S. Uyeto, Tetrahedron Letters [London] $1965,3473$.

${ }^{4}$ J. C. Sheehan and E. J. Corey, Org. Reactions 9, 388 [1957].

5 J. C. Sheehan and A. K. Bose, J. Amer. chem. Soc. 72, 5158 [1950].
HAN and Bose was extended by CHATterjee and RAO, to systems in which the methine hydrogen atom was activated by groups other than two ester functions ${ }^{7-9}$. It was noted that the closely related intra-molecular alkylation of $\omega$-halogen-alkylmalonic esters ocurred in poor yields even in the presence of sodium ethoxide ${ }^{10}$, while the base of choice in the Bose $\mathrm{Sheehan}$ synthesis was triethylamine.

6 J. C. Sheehan and A. K. Bose, J. Amer. chem. Soc. 73, 1261 [1951].

7 B. G. Chatterjee, P. N. Moza, and S. K. Roy, J. org. Chemistry 28, 1418 [1963].

8 B. G. Chatterjee, V. V. Rao, and B. N. G. Mazumdar, J. org. Chemistry 30, 4101 [1965].

9 B. G. Chatterjee, V. V. Rao, S. K. Roy, and H. P. S. CHAwla, Tetrahedron [London] 23, 492 [1967].

10 H. N. Walborsky, J. Amer. chem. Soc. 71, 2941 [1949]. 